\title{
Parametric estimation of reference signal intensity in the quantification of amyloid-beta deposition: an ${ }^{18} \mathrm{~F}-\mathrm{AV}-45$ study
}

\author{
Min Wang ${ }^{1,2 \#}$, Zhuangzhi Yan ${ }^{2}$, Huiwei Zhang ${ }^{3 \#}$, Jiaying $\mathrm{Lu}^{3}$, Ling $\mathrm{Li}^{3}$, Jintai Yu ${ }^{4}$, Jian Wang ${ }^{4}$, Hiroshi Matsuda $^{5}$, \\ Chuantao Zuo ${ }^{3}$, Jiehui Jiang ${ }^{1,2}$; the Alzheimer's Disease Neuroimaging Initiative \\ ${ }^{1}$ Shanghai Institute for Advanced Communication and Data Science, ${ }^{2}$ Institute of Biomedical Engineering, School of Communication and \\ Information Engineering, Shanghai University, Shanghai, China; ${ }^{3}$ PET Center, Huashan Hospital, ${ }^{4}$ Department of Neurology and Institute of \\ Neurology, Huashan Hospital, Shanghai Medical College, Fudan University, Shanghai, China; ${ }^{5}$ Integrative Brain Imaging Center, National Center \\ of Neurology and Psychiatry, Kodaira, Tokyo, Japan
}

\#These authors contributed equally to this work.

Correspondence to: Jiehui Jiang. Shanghai Institute for Advanced Communication and Data Science, Shanghai University, 99 Shangda Road, Shanghai 200444. China. Email: jiangjiehui@shu.edu.cn; Chuantao Zuo. PET Center, Department of Nuclear Medicine, Huashan Hospital, Fudan University, 518 Wuzhong Dong Road, Shanghai 201103, China. Email: zuochuantao@fudan.edu.cn.

Background: Positron emission tomography (PET) with the radiotracer florbetapir $\left({ }^{18} \mathrm{~F}-\mathrm{AV}-45\right)$ allows the pathophysiology of Alzheimer's disease $(\mathrm{AD})$ to be tracked in vivo. The semi-quantification of amyloidbeta $(A \beta)$ has been extensively evaluated with the standardized uptake value ratio (SUVR) but is susceptible to disturbance from the candidate reference region and the partial volume effect (PVE). In the present study, we applied the parametric estimation of reference signal intensity (PERSI) method to ${ }^{18} \mathrm{~F}$-AV-45 PET images for intensity normalization.

Methods: We enrolled 479 people with ${ }^{18} \mathrm{~F}-\mathrm{AV}-45$ images from the Alzheimer's Disease Neuroimaging Initiative database: 261 healthy controls (HCs), 102 patients with mild cognitive impairment (MCI), and $116 \mathrm{AD}$ patients. We used white matter post-processed by PERSI (PERSI-WM) as the reference region and compared our proposed method with the traditional method for semi-quantification. SUVRs were calculated for eight regions of interest: the frontal lobe, the parietal lobe, the temporal lobe, the occipital lobe, the anterior cingulate cortex, the posterior cingulate cortex, the precuneus, and the global cortex. The SUVRs derived from PERSI-WM and other reference regions were evaluated by effect size and receiver-operator characteristic curve analyses.

Results: The SUVRs derived from PERSI-WM showed significantly higher trace retention in the frontal, parietal, temporal, and occipital lobes, as well as in the anterior cingulate, posterior cingulate, precuneus, and global cortex in the AD A $\beta$-positive (+) group (mean: $+43.3 \% \pm 5.4 \%, \mathrm{P}<0.01$ ) and MCI A $\beta+$ group (mean: $+29.6 \% \pm 5.3 \%, \mathrm{P}<0.01)$. For the global cortex, PERSI-WM had the greatest Cohen's $\mathrm{d}$ effect size compared with the $\mathrm{HC} \mathrm{A} \beta$-negative (-) group ( $\mathrm{AD} \mathrm{A} \beta+$ and $\mathrm{MCI} \mathrm{A} \beta+$ : 3.02, $\mathrm{AD} \mathrm{A} \beta+$ : 3.56, MCI $\mathrm{A} \beta+: 2.34)$, and the highest area under the curve (AUC) between the $\mathrm{HC} A \beta$ - and $\mathrm{AD} A \beta+$ groups (AUC: $0.983,95 \%$ confidence interval: 0.978-0.998).

Conclusions: PERSI-WM could mitigate the influence of PVE and improve the semi-quantification of ${ }^{18} \mathrm{~F}-\mathrm{AV}-45$ images; therefore, it could be used for large-scale clinical application in the nuclear medicine domain.

^ ORCID: Min Wang, 0000-0002-3436-4246; Jiehui Jiang, 0000-0003-4948-3683. 


\begin{abstract}
Keywords: Myloid-beta (A $\beta$ ); florbetapir positron emission tomography; quantification; standardized uptake value ratio (SUVR); Alzheimer's disease (AD); parametric estimation of reference signal intensity (PERSI)
\end{abstract}

Submitted Jan 14, 2020. Accepted for publication Sep 07, 2020.

doi: $10.21037 /$ qims-20-110

View this article at: http://dx.doi.org/10.21037/qims-20-110

\section{Introduction}

Positron emission tomography (PET) using relevant biochemical markers can be used to visualize and characterize the pathophysiology of neurodegenerative diseases. Alzheimer's disease (AD) is a slowly progressive neurodegenerative brain disease and the most common cause of dementia (1). The pathological hallmarks of $\mathrm{AD}$ involve the co-occurrence of two aggregated proteins: amyloid-beta $(\mathrm{A} \beta)$, which deposits as amyloid plaques, and hyperphosphorylated forms of tau, which assemble into neurofibrillary tangles (NFTs) $(2,3)$. Studies have suggested that $A \beta$ accumulates decades before the onset of cognitive symptoms, and initiates or accelerates antecedent tauopathy in $\mathrm{AD}$ (4-6). Therefore, $\mathrm{A} \beta$ deposition can be used for the diagnosis of early-onset $\mathrm{AD}$ and clinical intervention.

PET with radionuclide-labeled agents targeting amyloid plaques provides a non-invasive method for tracking $A \beta$ accumulation in the brain in vivo (7). The 2011 National Institute on Aging and Alzheimer's Association guidelines for AD were updated in 2018 (8). In these guidelines, $\mathrm{AD}$ is defined as a continuous process in biomarker domains, including $A \beta$ deposition, pathological tau, and neurodegeneration. This is one of the accepted standards for the characterization of dementia and mild cognitive impairment (MCI), as well as for those subjects without cognitive impairment. In this system, amyloid plaques and NFTs are considered to define $\mathrm{AD}$ as a unique neurodegenerative disease among different various disorders that can lead to dementia. A $\beta$ biomarkers can be used to determine if an individual is in the " $\mathrm{AD}$ continuum". Therefore, the accurate identification and quantification of $\mathrm{A} \beta$ enable accurate characterization and understanding of the sequence of events that lead to cognitive impairment associated with $\mathrm{AD}$.

In recent decades, several research teams have developed a series of amyloid ligands to trace amyloid plaquerelated binding signals in $\mathrm{AD}$ brains [e.g., ${ }^{11} \mathrm{C}$-Pittsburgh compound B $\left({ }^{11} \mathrm{C}\right.$-PiB) $){ }^{18} \mathrm{~F}$-florbetaben, ${ }^{18} \mathrm{~F}$-flutemetamol, florbetapir $\left.\left({ }^{18} \mathrm{~F}-\mathrm{AV}-45\right)\right]$ labeled with appropriate PET nuclides $\left({ }^{11} \mathrm{C}\right.$ or $\left.{ }^{18} \mathrm{~F}\right)(9-12) .{ }^{18} \mathrm{~F}-\mathrm{AV}-45$ is currently a common radiotracer for PET, and is known for its fast kinetics, highly specific binding to amyloid plaques, and its long half-life (10). Studies have shown that ${ }^{18} \mathrm{~F}-\mathrm{AV}-45$ uptake in $\mathrm{AD}$ patients is significantly higher than that in healthy controls (HCs) or in patients with MCI $(13,14)$. In early $\mathrm{MCI}, \mathrm{A} \beta$ deposition starts in the precuneus region, as well as in the frontal and temporal regions. However, significant negative correlations between Mini-Mental State Examination (MMSE) score and standardized uptake value ratio (SUVR) from ${ }^{18} \mathrm{~F}-\mathrm{AV}-45$-labeled images have not been reported in AD patients (14). Some longitudinal studies have described unexpected decreases in SUVR in $\mathrm{AD}$ when applying a cerebellar, rather than a subcortical, white matter (WM) reference region $(15,16)$. These observations might be because inappropriate semi-quantitative analyses lead to underestimation of the severity of $A \beta$ deposition.

Consequently, semi-quantitative analyses of $A \beta$ using PET are crucial for the investigation of imaging biomarkers in the progression of $\mathrm{AD}$. The SUVR of regions of interest (ROIs) is an alternative measurement derived from the ratio of the mean $A \beta$ concentration relative to that in the reference region. Scholars studying the semi-quantification of $A \beta$ often select the whole cerebellum or cerebellar gray matter as the reference region (17). However, these cerebellar regions are susceptible to noise or adjacent cortical tissues that show specific binding. In particular, cerebellar signals are collected at the edge of the scanner's field of view, where sensitivity is lower, and the low position of the cerebellum may cause artifacts via truncation or attenuation correction of images (15). Longitudinal studies on AD have suggested that using subcortical $W M$ as a reference region for count normalization may facilitate more accurate tracing of $A \beta$ accumulation $(13,15,18,19)$. WM is a larger region and can represent the average uptake value of signal intensity, potentially leading to less noise. WM measurements may also be more resistant to small degrees of misregistration during image quantification (19). Furthermore, the partial volume effect (PVE) causes an apparent spillover or cross-contamination 
of counts between adjacent structures $(20,21)$. Therefore, determining the optimal reference region and eliminating PVE present challenges.

A series of PVE correction methods was recently presented to address concerns regarding the semiquantification of $\mathrm{A} \beta$ using PET imaging $(15,19,22)$. One of the methods of particular interest was the parametric estimation of reference signal intensity (PERSI) proposed by Southekal et al. for the patterns of tau accumulation based on flortaucipir (23). PERSI is an innovative and data-driven approach to count normalization that identifies a subjectspecific reference region instead of the traditional fixed atlas-based reference region. The underlying hypothesis of PERSI is that the reference region in flortaucipir images includes two major categories of voxels: voxels with contamination and voxels with non-specific binding. PERSI aims to identify appropriate non-specific binding voxels that have a lower intensity than other contaminated voxels. This approach uses the signal-intensity histogram of reference region to fit a bimodal Gaussian distribution to reject contaminated voxels in higher-intensity peak and then form a specific reference region. Multiple PVE correction algorithms typically involve estimating a better approximation of "true" tracer uptake from a mixture of signals by eroding or shrinking the reference region $(20,21)$. These methods correct the effects of spillover via PET itself or detailed anatomic information [e.g., the WM region from structural magnetic resonance imaging (MRI)] $(24,25)$. Recent studies have suggested that complicated procedures, restricted image demands, and the absence of sensitivity to noise prevent the complete elimination of PVE $(19,23)$. Therefore, the count-normalization strategy of PERSI has the potential to generate reliable and intercomparable SUVRs for ${ }^{18} \mathrm{~F}-\mathrm{AV}-45$ PET imaging.

In the present study, we investigated the spatial extent of $\mathrm{A} \beta$ in vivo for ${ }^{18} \mathrm{~F}-\mathrm{AV}-45$ PET using the PERSI method in HCs, as well as for patients with MCI and AD. We evaluated the count-normalization performance of PERSI using WM as a reference region in different groups with amyloid plaque accumulation. The reliability of our proposed method was evaluated using a longitudinal dataset.

\section{Methods}

\section{Participants}

The ${ }^{18} \mathrm{~F}-\mathrm{AV}-45$ PET and T1-weighted MRI data used in the present study were from the Alzheimer's Disease
Neuroimaging Initiative (ADNI) database (http://adni. loni.usc.edu/) (26). The ADNI was launched in 2003 as a public-private partnership led by principal investigator Michael W. Weiner. The primary aim of this project was to test whether serial MRI, PET, and other biologic markers, along with clinical and neuropsychological assessments, can be combined to measure the progression of MCI and early $\mathrm{AD}$.

The present study enrolled 479 people: $261 \mathrm{HCs}$, $102 \mathrm{MCI}$ patients, and $116 \mathrm{AD}$ patients. All participants underwent ${ }^{18} \mathrm{~F}-\mathrm{AV}-45$ PET and structural MRI imaging at baseline. Furthermore, we stratified each group into $\mathrm{A} \beta$ positive $(+)$ and negative (-) via the cerebral-to-whole cerebellum A $\beta$ SUVR provided by the ADNI, with a threshold of 1.18 , according to previously published autopsy-validated findings (15). In total, $62 \mathrm{HCs}, 62 \mathrm{MCI}$ patients, and $99 \mathrm{AD}$ patients were classified as $\mathrm{A} \beta+$, and 199 HCs, 40 MCI patients, and 17 AD patients were classified as A $\beta-$. Fourteen of $116 \mathrm{AD}$ patients had 2 followup scans with ${ }^{18} \mathrm{~F}-\mathrm{AV}-45$ PET images. Each individual was assessed using extensive neuropsychological and cognitive examinations: Montreal Cognitive Assessment (MOCA), Clinical Dementia Rating-Sum of Boxes (CDR-SB), and MMSE scores.

Detailed eligibility criteria for these cross-sectional data are shown in Figure S1. The diagnosis-specific exclusion and inclusion criteria for the HC, MCI, and AD groups are stated in the ADNI dataset (http://adni.loni. usc.edu/methods/documents/). The study protocol was approved by the Institutional Review Board of the ADNI and all participating institutions. Each participant gave written informed consent. The demographic and clinical characteristics of all participants are summarized in Table 1.

\section{Acquisition and preprocessing of images}

The original ${ }^{18} \mathrm{~F}-\mathrm{AV}-45 \mathrm{PET}$ and $\mathrm{T} 1$-weighted structural MRI scans were acquired from the ADNI database. Dynamic 3-dimensional (3D) PET images of $4 \times 5 \mathrm{~min}$ frames were acquired $50 \mathrm{~min}$ after bolus injection of approximately $370 \pm 37 \mathrm{MBq}{ }^{18} \mathrm{~F}-\mathrm{AV}-45$. PET images were reconstructed specific to the scanners, with different iterations and subsets. All participants also underwent volumetric T1-weighted MRI using 1.5-T or 3-T systems. PET and MRI data were acquired according to standardized ADNI protocols (adni.loni.usc.edu/methods/documents/).

For dynamic PET scans, 2-4 frames were rigidly realigned to the first frame for motion correction and then 
Table 1 Clinical ratings and demographic characteristics of all participants

\begin{tabular}{|c|c|c|c|c|c|c|c|c|c|}
\hline Characteristics & \multicolumn{3}{|c|}{ HCs (n=261) } & \multicolumn{3}{|c|}{ MCl patients $(n=102)$} & \multicolumn{3}{|c|}{ AD patients $(n=116)$} \\
\hline Sex (male/female) & $20 / 42$ & $86 / 113$ & 0.166 & $34 / 28$ & $28 / 12$ & 0.185 & $51 / 48$ & $13 / 4$ & 0.099 \\
\hline Age (years) & $76.5 \pm 5.61$ & $72.3 \pm 6.04$ & $<0.001$ & $74.8 \pm 6.03$ & $75.3 \pm 6.17$ & 0.759 & $73.6 \pm 8.07$ & $75.6 \pm 7.47$ & 0.335 \\
\hline Education (years) & $16.3 \pm 2.68$ & $16.6 \pm 2.48$ & 0.462 & $16.1 \pm 2.67$ & $15.9 \pm 2.97$ & 0.796 & $15.7 \pm 2.66$ & $15.8 \pm 2.75$ & 0.889 \\
\hline MOCA & $25.4 \pm 2.64$ & $26.3 \pm 2.54$ & 0.022 & $18.5 \pm 5.29$ & $21.8 \pm 4.71$ & 0.001 & $15.5 \pm 6.09$ & $16.8 \pm 4.85$ & 0.363 \\
\hline CDR-SB & $0(0,0)$ & $0(0,0)$ & 0.180 & $4.5(1.5,6.0)$ & $1.5(0.5,2.125)$ & $<0.001$ & $4.5(4.5,6.0)$ & $4.5(3.25,4.5)$ & 0.674 \\
\hline APOE $\varepsilon 4$ positivity (\%) & 53.2 & 22.1 & $<0.001$ & 66.1 & 14.3 & $<0.001$ & 74.7 & 23.5 & $<0.001$ \\
\hline
\end{tabular}

Data are reported as mean \pm standard deviation or median (interquartile range). A $\beta$, amyloid-beta; $A D$, Alzheimer's disease; HCs, healthy controls; APOE $\varepsilon 4$ positivity, positive rate for the presence of at least $1 \varepsilon 4$ apolipoprotein E; CDR-SB, Clinical Dementia Rating-Sum of Boxes; MCI, mild cognitive impairment; MOCA, Montreal Cognitive Assessment; MMSE, Mini-Mental State Examination; +, positive; -, negative.

averaged. An individual PET image was then registered to the corresponding T1-weighted magnetic resonance image using rigid-body registration, and the magnetic resonance image was spatially normalized to the Montreal Neurological Institute (MNI) atlas space using the MNI152 2-mm T1-weighted MRI template (27). The PET image was spatially transformed onto the MNI152 space via MRI transformation parameters. Finally, the normalized PET images were spatially smoothed with an 8-mm full-width at half maximum (FWHM) Gaussian kernel over a 3D space to increase the signal-to-noise ratio. All preprocess procedures were performed with statistical parametric mapping software (SPM12; Wellcome Department of Imaging Neuroscience, London, UK) implemented in MATLAB 2014b (Mathworks, Sherborn, MA, US).

\section{PERSI approach}

${ }^{18} \mathrm{~F}-\mathrm{AV}-45$ images from all participants were analyzed quantitatively using the PERSI method to identify a subject-specific reference region for $A \beta$ quantification. We used a reference region-corrected approach analogous to that described previously (23), in which an automated computerized method was applied to the WM region to remove the influence of voxel contamination. As a datadriven method, PERSI is committed to identifying the nonspecific ${ }^{18} \mathrm{~F}-\mathrm{AV}-45$ binding region as the reference region using a signal-intensity histogram of the region (Figure 1).

In this context, we used the WM region as the masked region. For the WM region, we applied the segmentation algorithm in FMRIB Software Library (FSL) to the MNI152 atlas to generate the initial WM mask. Preprocessed ${ }^{18} \mathrm{~F}-\mathrm{AV}-45$ PET images were masked with this WM region, and the voxel sets of the masked region were estimated with a histogram. An individual histogram was fitted using a bimodal Gaussian distribution, in which the location and sigma value of two peaks were estimated by a non-linear least-squares algorithm as the following mathematical equation:

$$
\text { model }_{\text {hist }}=a_{1} \times e^{-\frac{\left(x-\mu_{1}\right)^{2}}{\sigma_{1}^{2}}}+a_{2} \times e^{-\frac{\left(x-\mu_{2}\right)^{2}}{\sigma_{2}^{2}}}
$$

model $_{\text {hist }}$ represents the voxel histogram set in the WM region. The first Gaussian peak was regarded as having non-specific binding intensity, and its peak center location $\left(\mu_{1}\right)$ and sigma $\left(\sigma_{1}\right)$ were used for reference analyses. The WM region with the voxel intensity within the range was a subject-specific reference region:

$$
\text { Range }=\mu_{1} \pm \mathrm{FWHM} / 2 \approx \mu_{1} \pm 1.178 \times \sigma_{1}
$$

The second Gaussian peak was considered to be contaminated by counts from adjacent cortical tissues with higher specific binding, and the voxels in this range were removed from the WM region. After obtaining the subjectspecific reference region, we padded this region to avoid the holes caused by noise. As a result, the eventual PERSIWM masks used in the present study were still a part of the previous WM masks. 


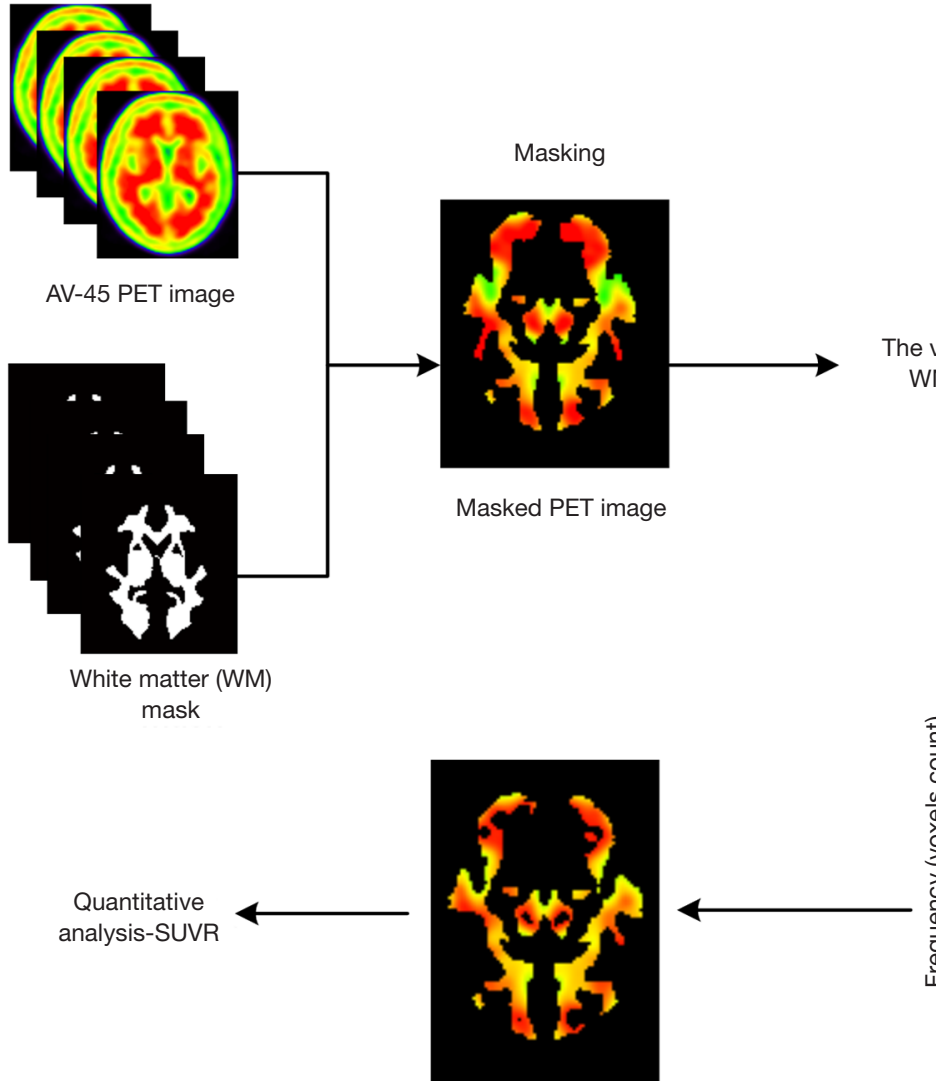

The subject-specific WM

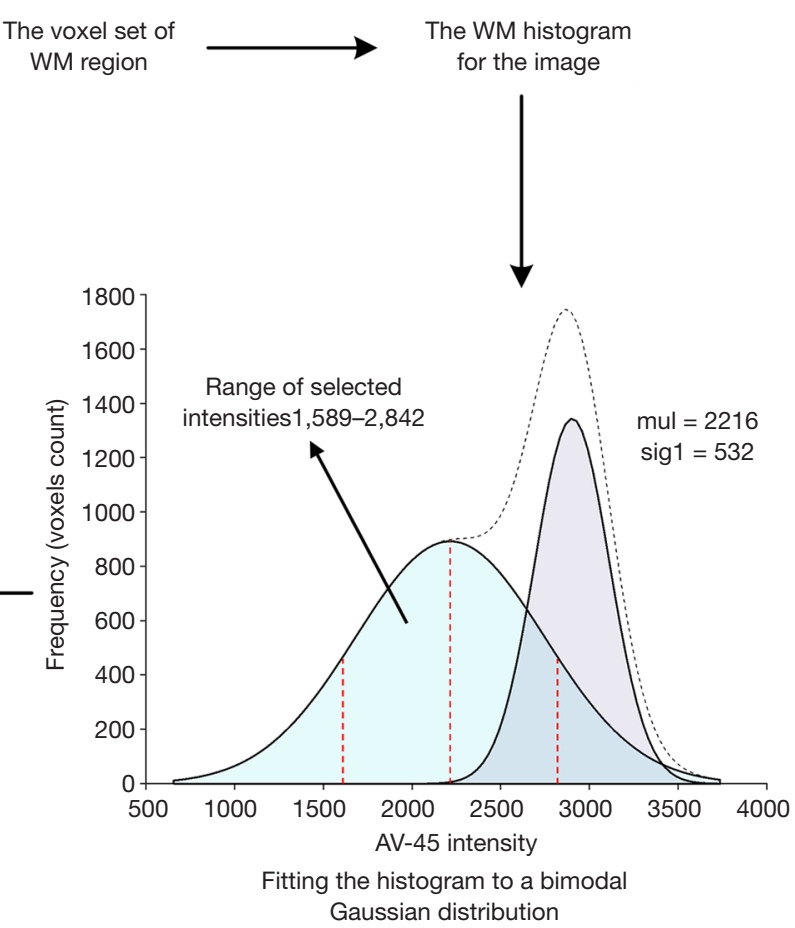

Figure 1 Flowchart of the parametric estimation of reference signal intensity approach. Preprocessed florbetapir positron emission tomography image was first masked using a white matter (WM) template to generate the voxel set. A histogram of this voxel set in WM was then fitted to a bimodal Gaussian distribution, as shown in the bottom right panel in which the dotted line represents the real histogram curve. Lower intensity peak, as shown the left peak ( $\mathrm{mul}=2,216$, sigl $=532$ ) was used to measure the intensity range [mul \pm full-width at half maximum/2 ( $\approx 1.178$ sigl); 1,589-2,842] in the WM image to generate a subject-specific WM region as a reference region. Generated reference region was further used for semi-quantitative standardized uptake value ratio analyses.

\section{SUVR analyses}

To evaluate the quantitative performance of PERSI in A $\beta$ radiotracer images, we used three additional reference regions to generate voxel-wise ${ }^{18} \mathrm{~F}-\mathrm{AV}-45$ SUVR images: the whole cerebellum, MNI atlas-based WM, and subjectspecific WM. The whole cerebellum was derived from the Automated Anatomical Labeling template (28). The MNI atlas-based WM region was obtained by segmentation of the MNI152 atlas. For the subject-specific WM region, the segmentation probabilistic WM image from individual magnetic resonance images had a threshold of 0.5 (23). The
MNI atlas-based and subject-specific WM regions were processed by a composite mask and 3-voxel erosion box to mitigate the PVE. The composite mask of the cerebral cortex, brain stem, and cerebellum was applied to these WM masks to remove the associated voxels susceptible to PVE spillover.

Eight candidate target regions were predefined, and the SUVRs in these regions were measured using reference regions. These predefined ROIs (frontal lobe, parietal lobe, temporal lobe, occipital lobe, anterior cingulate, posterior cingulate, precuneus, and global cortex) have been reported to affect the progression of neurodegeneration in $\mathrm{AD}$ by 
A $\beta$ deposition $(13,29)$. Therefore, we generated bilateral ROIs via the brain parcellation atlas in the MNI space. The SUVRs of these ROIs were obtained as the count ratio by averaging the voxel-wise SUVR image in a specific targeted region.

\section{Statistical analyses}

Clinical and demographic characteristics were compared between groups using one-way analysis of variance (ANOVA; age, education, MMSE, MOCA, and CDR-SB scores) or the $\chi^{2}$-test [sex and apolipoprotein E (APOE) $\varepsilon 4$ positivity]. Dunnett's $t$-method with correction of the $\mathrm{P}$ value was used to adjust for multiple comparisons in the ANOVA. To evaluate the quantitative performance of PERSI-WM and other traditional reference regions, we implemented the effect size with standardized Cohen's $\mathrm{d}$ to measure the between-group differences of SUVRs. We applied receiver operating characteristic (ROC) curve analyses to each regional SUVR to evaluate discrimination between diagnostic groups, and area under the curve (AUC) values as a measurable indicator. Correlations between regional SUVRs and cognitive assessments were evaluated using Spearman's coefficient, and the $\mathrm{P}$ value was corrected using the Bonferroni correction method. Pairwise comparisons of correlations were done using bootstrapping of correlation coefficient pairs with 1,000 permutations. The ${ }^{18} \mathrm{~F}-\mathrm{AV}-45$ PET image was mapped in the smoothed 3D template across each group using the BrainNet Viewer toolbox (version 1.62; www.nitrc.org/projects/bnv/) (30). $\mathrm{P}<0.05$ (two-tailed) was considered statistically significant. Statistical analyses were performed using R-3.5.1 (www. r-project.org/).

\section{Results}

\section{Participants}

The demographic information and clinical characteristics of the different diagnostic groups are detailed in Table 1. Table $\mathrm{S} 1$ shows the demographic and clinical characteristics for the 14 longitudinal AD patients with ${ }^{18} \mathrm{~F}-\mathrm{AV}-45$ PET images $(2.23 \pm 0.83$ years). The mean age differed significantly between the $\mathrm{HC} A \beta+$ and $\mathrm{HC} A \beta$ - subgroups $(\mathrm{P}<0.001)$. However, no significant differences were found in terms of sex or years of education between $A \beta+$ and $A \beta-$ status in any of the groups $(\mathrm{P}>0.05$ for all). As expected, $A \beta+$ participants were more likely to have APOE $\varepsilon 4$ positivity
(AD $\mathrm{A} \beta+$ : $74.7 \%$, MCI $\mathrm{A} \beta+$ : 66.1\%, $\mathrm{HC} \mathrm{A} \beta+: 22.1 \%$ ) than those who were $\mathrm{A} \beta-\left(\mathrm{P}<0.001, \chi^{2}\right.$-test $)$. Upon completion of all neuropsychological assessments (MMSE, MOCA, and CDR-SB scores), individuals with cognitive impairment performed significantly worse than the HCs $(\mathrm{P}<0.05$ for all; ANOVA with Dunnett's multiple comparison). The longitudinal AD patients had significant changes in cognitive assessments (all $\mathrm{P}<0.05,1$-sample $t$-test).

\section{Semi-quantification of ${ }^{18} \mathrm{~F}-A V-45$ imaging}

Figure 2 shows histograms with different WM signal intensities derived from the PERSI method; the higher peak of HCs was not significant. For individuals with cognitive decline, the higher peak was more prominent, which suggested that some voxels were contaminated by adjacent cortical tissues with ${ }^{18} \mathrm{~F}-\mathrm{AV}-45$ uptake. The PERSI approach could be used to identify and remove these contaminated voxels from the WM reference region. We summarized the size of the reference regions (Table 2). The average PERSI-WM region for $\mathrm{AD}$ cases had the smallest volume size relative to those in other groups $(\mathrm{P}<0.05) ; \mathrm{A} \beta+$ patients showed a significant decrease compared with $A \beta$ patients $(\mathrm{P}<0.001)$.

To explore the difference in quantitative efficacy between the PERSI-WM region and other traditional reference regions, we examined ${ }^{18} \mathrm{~F}-\mathrm{AV}-45$ SUVRs within each group of predefined ROIs. As shown in Figure 3, compared with HC A $\beta-$, the SUVRs derived from PERSI-WM showed significantly higher trace retention in the frontal, parietal, temporal, occipital, anterior cingulate, and posterior cingulate, precuneus, and global cortex in the $\mathrm{AD} A \beta+$ group (mean: $+43.3 \% \pm 5.4 \%, \mathrm{P}<0.01$ ) and $\mathrm{MCI} \mathrm{A} \beta+$ group (mean: $+29.6 \% \pm 5.3 \%, \mathrm{P}<0.01$ ). Furthermore, the PERSIWM method could accurately track the $\mathrm{A} \beta$ accumulation of predefined ROIs between the MCI and AD groups relative to other reference regions $(\mathrm{P}<0.05$ for all, Dunnett's multiple comparison) (Table 3). These phenomena indicate the superiority of the PERSI-WM method for $\mathrm{A} \beta$ quantification, especially in individuals with cognitive decline.

ROC and effect size were analyzed to evaluate the diagnostic ability of PERSI compared with other traditional reference regions (Table 3). PERSI-WM had a greater effect size and AUC in ROIs compared with other reference regions. For the global cortex, PERSI-WM had the greatest Cohen's d effect size compared to HC A $\beta-(\mathrm{AD} A \beta+$ and MCI $\mathrm{A} \beta+$ : 3.02, $\mathrm{AD} \mathrm{A} \beta+$ : 3.56, MCI $\mathrm{A} \beta+$ : 2.34) and the highest 

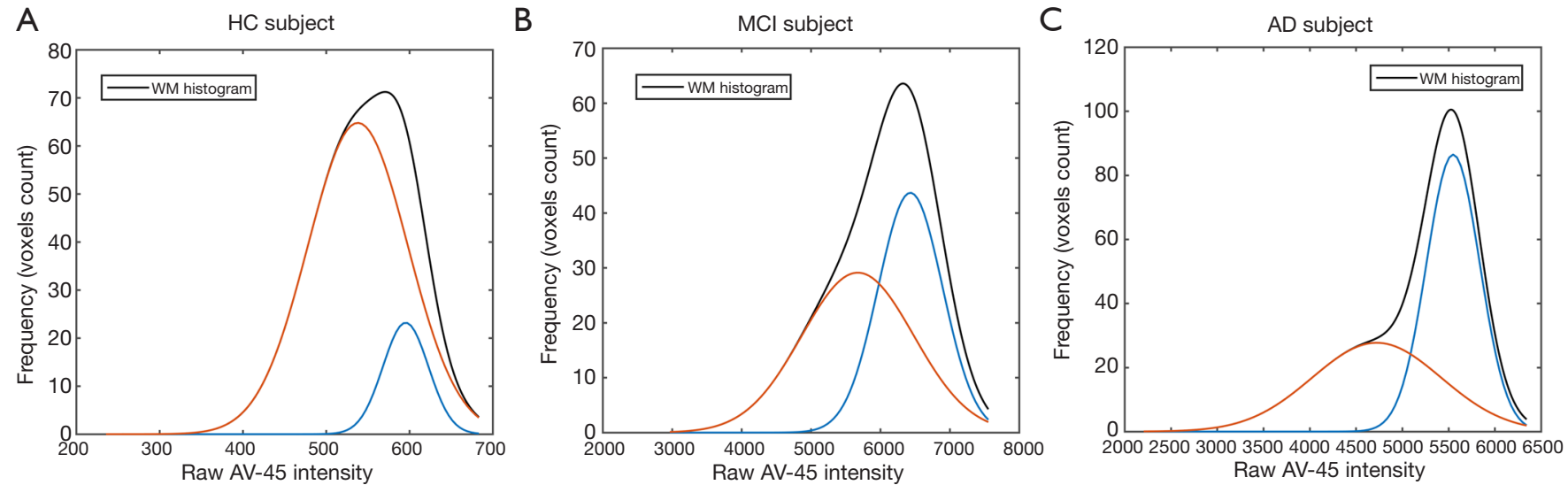

Figure 2 Typical white matter (WM) signal-intensity histograms for representative participants categorized as healthy control (HC) amyloid-beta $(\mathrm{A} \beta)$ negative (A, 84-year-old male), and mild cognitive impairment amyloid-beta positive (A $\beta+)(\mathrm{B}, 73$-year-old male), and Alzheimer's disease $\mathrm{A} \beta+(\mathrm{C}, 56$-year-old female). Black line represents the real histogram in the WM region, red line represents lower intensity signal, and blue line represents higher intensity signal. Second peak is not significant for the HC. For patients with cognitive decline, some contamination voxels in the WM region are indicated by the blue peak.

Table 2 Reference region sizes of the different groups

\begin{tabular}{lc}
\hline Reference region & Volume size $(\mathrm{mL})^{\dagger}$ \\
\hline Whole cerebellum & 177.7 \\
MNI atlas-based WM & 88.2 \\
Average subject-specific WM & $60.6 \pm 11.0$ \\
Average PERSI-WM & \\
HC & $121.5 \pm 22.8$ \\
MCI & $76.6 \pm 13.4$ \\
AD & $72.1 \pm 17.6$ \\
$A \beta-$ & $110.3 \pm 28.9$ \\
$A \beta+$ & $87.6 \pm 28.8$ \\
\hline
\end{tabular}

${ }^{\dagger}, 2^{*} 2^{*} 2 \mathrm{~mm}$ voxels. AD, Alzheimer's disease; $A \beta$, amyloidbeta; $\mathrm{HC}$, healthy control; $\mathrm{MCl}$, mild cognitive impairment; $\mathrm{MNI}$, Montreal Neurological Institute; PERSI, parametric estimation of reference signal intensity; WM, white matter; +, positive; -, negative.

AUC between the HC A $\beta$ - and AD A $\beta+$ groups (AUC: 0.983 , 95\% confidence interval: 0.978-0.998). Estimates of the effect size and AUC indicated that the PERSI-WM approach increased between-group differences compared to those in the whole cerebellum (Table 3, Tables S2-S8).

To explore the influence on count normalization using the PERSI approach, we evaluated linear regression across two normalized methods (Figure 4). Compared to other reference region methods using WM, PERSI-WM generated an increase in the measurement of ${ }^{18} \mathrm{~F}-\mathrm{AV}-45$ trace retention of the global cortex (subject-specific WM slope: 1.05, MNI atlas WM slope: 1.09). The SUVR of the global cortex using PERSI-WM, especially in AD patients, increased significantly compared to that using traditional WM reference regions $(\mathrm{P}<0.001$ for all), whereas a negligible increase was documented in the HCs (subjectspecific WM: $\mathrm{P}=0.278, \mathrm{MNI}$ atlas WM: $\mathrm{P}=0.371)$. These phenomena suggested that PERSI-WM could aid in the estimation of the actual $A \beta$ signal in adjacent structures or off-targeted binding regions in patients with cognitive decline. Similar findings were observed in the other candidate ROIs. Figure 4 shows that PERSI-WM could be a powerful semi-quantitative method for reducing PVE in the measurement of $\mathrm{A} \beta$ accumulation with ${ }^{18} \mathrm{~F}-\mathrm{AV}-45$ PET imaging.

The longitudinal dataset was used to validate the effectiveness of our proposed method. We employed PERSI-WM and other reference regions to measure SUVRs at both baseline and the follow-up PET scans. Figure 5 shows cortical ${ }^{18} \mathrm{~F}-\mathrm{AV}-45$ SUVRs in the baseline and followup scans of $14 \mathrm{AD}$ participants. The results indicate that the global cortical SUVR with PERSI-WM appears less variable and has a paradoxical decrease compared with other methods. Moreover, the changes in the global cortical SUVR with PERSI-WM were significantly correlated with the changes in cognitive assessments (MMSE: $r=-0.343$, 

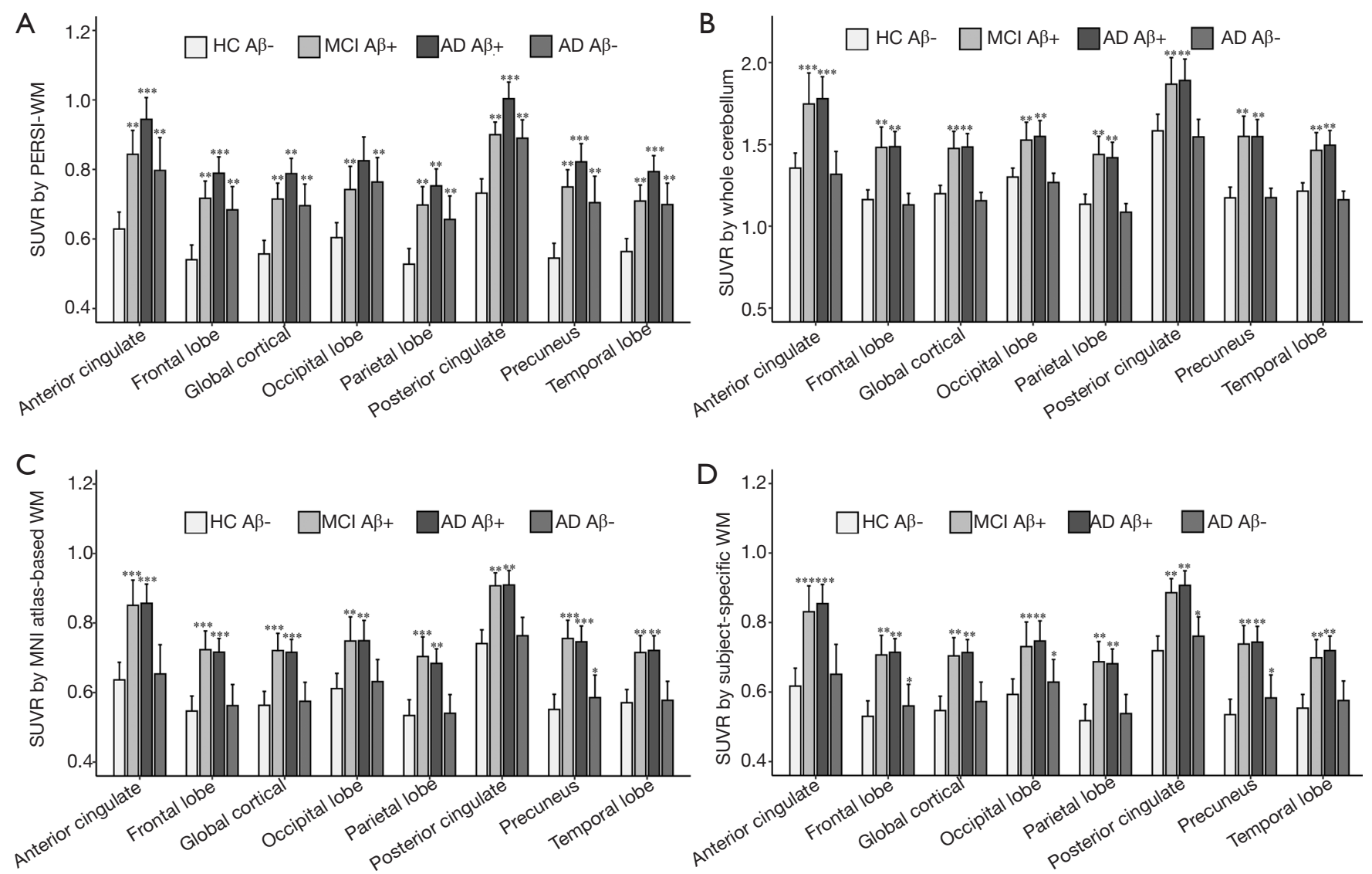

Figure 3 Global and regional florbetapir standardized uptake value ratio (SUVR) count normalization from parametric estimation of reference signal intensity: white matter (WM) (A), whole cerebellum (B), MNI atlas-based WM (C) and subject-specific WM region (D) for four diagnosed groups (HC A $\beta-$, MCI A $\beta+, A D A \beta+$, and AD A $\beta-$ ). Panel indicates the mean group SUVR ( \pm standard deviation) across predefined regions of interest. PERSI, parametric estimation of reference signal intensity; HC, health control; MNI, Montreal Neurological Institute; $\mathrm{A} \beta$, amyloid-beta; $\mathrm{AD}$, Alzheimer's disease; $\mathrm{MCI}$, mild cognitive impairment.

$\mathrm{P}=0.02$ ). Similar findings could be observed in other brain regions (Figure S2), indicating that PERSI-WM could be useful for $\mathrm{A} \beta$ quantification in longitudinal studies.

\section{${ }^{18} \mathrm{~F}-A V-45$ SUVRs and cognitive testing correlation}

To further validate the efficacy of our proposed method, we evaluated the correlation between the ${ }^{18} \mathrm{~F}-\mathrm{AV}-45 \mathrm{SUVR}$ and neuropsychological and cognitive test parameters (MMSE, MOCA, and CDR-SB) among A $\beta+$ patients with cognitive decline in ROIs. Table 4 shows the correlations between clinical cognitive scores and the SUVR in the MCI and $\mathrm{AD} A \beta+$ group in the global cortex using Spearman's correlation coefficients. Tables S9-S15 show the results of other candidate regions. As expected, there were significant associations between higher ${ }^{18} \mathrm{~F}-\mathrm{AV}-45$ SUVRs and worse scores for cognitive scores. Of 161 patients with the global cortex as the ROI, CDR-SB scores were more strongly correlated with PERSI-WM count-normalized SUVRs $(r=0.451, \mathrm{P}<0.01)$ than SUVRs derived from other methods $(\mathrm{P}<0.05)$. Similar findings were observed for MOCA and MMSE scores.

\section{Discussion}

${ }^{18} \mathrm{~F}-\mathrm{AV}-45$ is an in vivo PET biomarker that can be used to measure $A \beta$ plaque accumulation in the brain. In the present study, we evaluated the count-normalization performance of PERSI based on WM for tracking $A \beta$ pathophysiology in $\mathrm{AD}$. The subject-specific WM reference 
Table 3 Diagnostic ability of 4 semi-quantitative methods in the global cortex

\begin{tabular}{|c|c|c|c|c|c|}
\hline Characteristics & Group & PERSI-WM & Subject-specific WM & MNI atlas-based WM & Whole cerebellum \\
\hline \multirow{4}{*}{ SUVR (global cortex) } & $\mathrm{MCl} A \beta+$ & $0.71 \pm 0.06$ & $0.70 \pm 0.10$ & $0.72 \pm 0.11$ & $1.47 \pm 0.15$ \\
\hline & $\mathrm{AD} A \beta+$ & $0.79 \pm 0.05$ & $0.71 \pm 0.08$ & $0.71 \pm 0.05$ & $1.48 \pm 0.12$ \\
\hline & $\mathrm{HCA} \beta+$ & $0.63 \pm 0.06$ & $0.62 \pm 0.09$ & $0.64 \pm 0.06$ & $1.42 \pm 0.12$ \\
\hline & $A D A \beta-$ & $0.69 \pm 0.09$ & $0.57 \pm 0.09$ & $0.57 \pm 0.08$ & $1.15 \pm 0.07$ \\
\hline \multirow[t]{3}{*}{ AUC } & $\mathrm{AD} \& \mathrm{MCl} A \beta+v s . \mathrm{HC} A \beta-$ & 0.981 & $0.965^{\star}$ & $0.962^{*}$ & 0.976 \\
\hline & $A D A \beta+v s . H C A \beta-$ & 0.983 & $0.973^{*}$ & $0.964^{*}$ & 0.981 \\
\hline & $\mathrm{MCl} A \beta+$ vs. $\mathrm{HC} \mathrm{A} \beta-$ & 0.979 & $0.954^{*}$ & $0.956^{*}$ & $0.957^{*}$ \\
\hline Effect size & $\mathrm{MCl} A \beta+$ vs. $\mathrm{HC} \mathrm{A} \beta-$ & 2.34 & 2.03 & 2.12 & 2.21 \\
\hline
\end{tabular}

*, $\mathrm{P}<0.05$, AUC comparison between PERSI-WM and other reference regions (DeLong test). Effect size is given as the standardized Cohen's d. AD, Alzheimer's disease; $A \beta$, amyloid-beta; AUC, area under the curve; CDR-SB, Clinical Dementia Rating-Sum of Boxes; HC, healthy control; $\mathrm{MCI}$, mild cognitive impairment; MMSE, Mini-Mental State Examination; MNI, Montreal Neurological Institute; MOCA, Montreal Cognitive Assessment; PERSI, parametric estimation of reference signal intensity; SUVR, standardized uptake value ratio; WM, white matter; +, positive; -, negative.
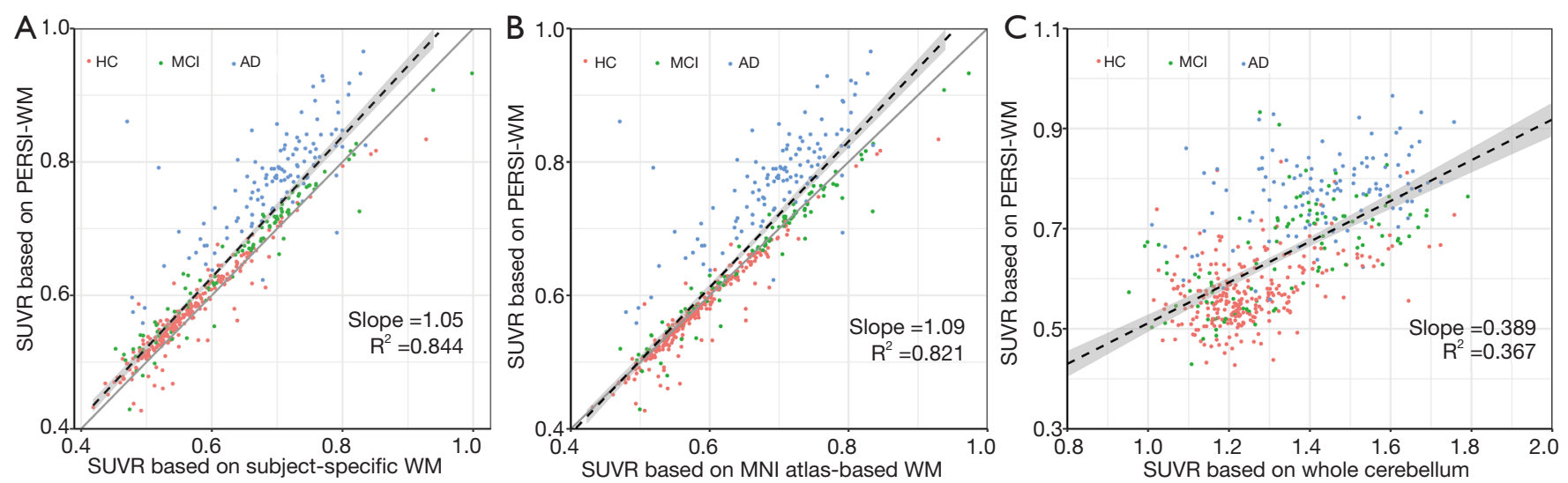

Figure 4 Scatterplots of florbetapir standardized uptake value ratio (SUVR) derived from different reference regions in the global cortex region. Red dots denote the SUVR of healthy control (HC) amyloid-beta (A $\beta$ )-negative participants, green dots denote the SUVR of mild cognitive impairment (MCI) A $\beta$-positive patients, and blue dots denote the SUVR of Alzheimer's disease (AD) A $\beta$-positive patients. Gray line represents the SUVR unchanged datum line (slope =1). PERSI, parametric estimation of reference signal intensity; WM, white matter; MNI, Montreal Neurological Institute.

region was estimated using the PERSI method and mitigated the PVE to generate intra- and inter-individual intercomparable SUVRs. We observed that $\mathrm{A} \beta$ deposition consistently occurred in anatomically ritualistic patterns, which is similar to findings with ${ }^{11} \mathrm{C}-\mathrm{PiB}$ PET (31-33). We also demonstrated that PERSI-WM had greater effect sizes and AUC values for the separation of diagnostic groups based on $\mathrm{A} \beta$ deposition in specific ROIs. Furthermore, correlations with cognitive test results were also higher in SUVRs by PERSI-WM. Our results suggested that 

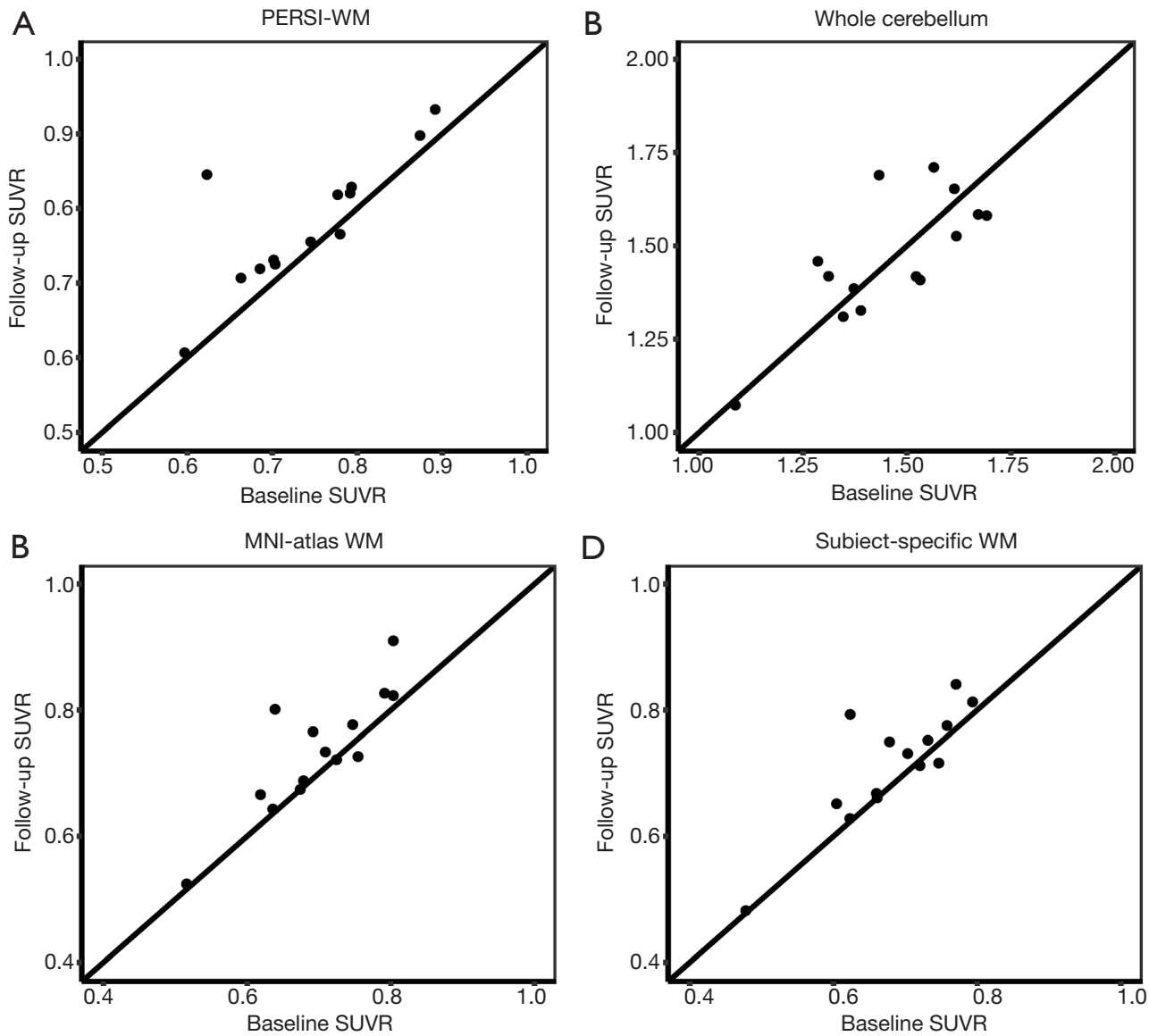

Figure 5 Scatterplots of global cortical florbetapir standardized uptake value ratios for the baseline and follow-up visits of 14 Alzheimer's disease patients using 4 different reference regions. SUVR, standardized uptake value ratio; MNI, Montreal Neurological Institute; WM, white matter.

Table 4 Correlations between clinical cognitive scores and standardized uptake value ratio in the mild cognitive impairment and Alzheimer's disease amyloid-beta-positive patient group

\begin{tabular}{|c|c|c|c|c|}
\hline Global cortex & PERSI-WM & Subject-specific WM & MNI atlas-based WM & Whole cerebellum \\
\hline MOCA & -0.384 & $-0.278^{\star \star}$ & $-0.288^{\star \star}$ & $-0.079^{\dagger}$ \\
\hline CDR-SB & 0.451 & $0.403^{*}$ & $0.407^{\star}$ & $0.229^{\star \star *}$ \\
\hline
\end{tabular}

${ }^{*}, \mathrm{P}<0.05$; ${ }^{* \star}, \mathrm{P}<0.01 ;{ }^{* \star \star}, \mathrm{P}<0.001$, pairwise comparisons of correlations between PERSI-WM and other reference regions. ${ }^{\dagger}$, insignificant correlation, $\mathrm{P}$ value with correction for multiple comparisons >0.05. CDR-SB, Clinical Dementia Rating-Sum of Boxes; MMSE, MiniMental State Examination; MNI, Montreal Neurological Institute; MOCA, Montreal Cognitive Assessment; PERSI, parametric estimation of reference signal intensity; WM, white matter.

PERSI was a relatively robust reference region correction method for amyloid images, and could be used to accurately measure $\mathrm{A} \beta$ changes over $\mathrm{AD}$ progression.

We used subcortical WM instead of the whole cerebellum as the reference region to measure the relative concentrations of ROIs in amyloid PET imaging. The ability of this reference region to identify diagnostic groups of SUVRs based on WM was greater than that of SUVRs 
based on other reference regions (Table 3). Conventionally, reference region known empirically for $\mathrm{A} \beta$ radiotracer is the whole cerebellum or cerebellum gray matter (34-36). These regions are expected to remain stable and free of $\mathrm{A} \beta$ binding during $\mathrm{AD}$ progression. However, their small size and proximity to the axial periphery of gray matter make these region sensitive to contamination from noise or other high-uptake voxels. The location of the whole cerebellum makes it susceptible to truncation. The subcortical WM region, compared with gray matter, is less sensitive to noise, has a lower clearance rate, as well as other reduced binding characteristics. Scholars have also noted that WM regions have lower variability than other reference regions according to longitudinal data $(15,16)$. Therefore, subcortical $W M$ is an alternative reference region for quantifying $\mathrm{A} \beta$ deposition, which has also been confirmed by recent studies $(15,19,37,38)$.

Additionally, we applied the PERSI method and WM reference region for count normalization in ${ }^{18} \mathrm{~F}-\mathrm{AV}-45$ imaging. The semi-quantitative performance of PERSIWM was superior to that of other reference regions for the stratification of diagnostic groups using traditional SUVRs (Figure 3, Table 3). A cross-sectional and longitudinal study suggested that traditional methods may not completely eliminate PVE because of some preprocessing steps, such as erosion and masking (23). A series of existing PVE correction methods can be used to overcome contamination in the reference region regarding $A \beta$ quantification $(19,20,39)$. However, the PERSI method can reduce potential contamination of WM regions when other PVE correction methods with complicated procedures are not performed (23). In the case of a lack of magnetic resonance scans, the PERSI method can still work. PERSI is designed to overcome the potential impact of PVE mentioned above and leverage the advantage of using the WM region. The PERSI method reduces direct contamination of the WM region without other preprocessing steps and is immune to differences in the uptake pattern (23). Therefore, in the present study, the PERSI method obtained more reliable and accurate semi-quantification results for ${ }^{18} \mathrm{~F}-\mathrm{AV}-45$ imaging, and it may be useful for clinical research and the diagnosis of patients with cognitive disorders.

The spatial distribution of ${ }^{18} \mathrm{~F}-\mathrm{AV}-45$ retention in the present study was consistent with that in previous AD neuropathological studies $(6,13,17,40-43)$. The differences in SUVR between the HCs and AD patients were more substantial with PERSI-WM as a reference region (Figure 3). A $\beta$ accumulation in the global cortex and anterior cingulate could be evaluated in $\mathrm{AD}$ patients, and the uptake patterns frequently encompassed large regions of the frontal lobe, parietal lobe, and posterior cingulate. $\mathrm{A} \beta$ accumulation in the eight predefined ROIs was highest for the AD patients, intermediate for the MCI patients, and lowest for the HCs. Other methods, such as the whole cerebellum and MNI atlas-based WM had negligible and undiscerning SUVR differences, especially in the MCI A $\beta+$ and $\mathrm{AD} A \beta+$ groups, whereas PERSI-WM surpassed other methods for diagnosing patients with cognitive decline with a different clinical diagnosis (Figures 3,4). Studies focusing on amyloid quantification have also suggested that using subcortical WM to normalize ${ }^{18} \mathrm{~F}-\mathrm{AV}-45$ images could more accurately track $A \beta$ changes and more closely reflect the "gold standard" volume of distribution differences between MCI and AD compared to other reference regions (13). We also used a subject-specific WM region and MNI atlasbased WM region to measure A $\beta$ SUVR and observed a similar result. The PERSI-WM region, compared with the two methods mentioned earlier, had satisfactory corrected volume counts for the effects of spillover in cognitive decline (MCI and $\mathrm{AD}$ ) patients and $\mathrm{A} \beta+$ patients. PERSIWM demonstrated excellent semi-quantitative ability and generated consistent SUVRs in candidate ROIs.

\section{Limitations}

The present study has some limitations. First, we used the atlas-WM mask to run PERSI procedures to enable broader applicability. The mask was obtained from the MNI152 template. The individual WM segmentations, derived from a structural magnetic resonance image, were also suitable for generating a subject-specific mask for each participant. We will investigate this aspect in future studies. Second, we used a traditional $8-\mathrm{mm}$ Gaussian kernel in the preprocessing step; however, different FWHM Gaussian windows may result in different PERSI results. It would be beneficial to investigate the influences of different Gaussian windows and choose an optimized window for PERSI-WM in future studies. Third, the PERSI-WM method proposed in the current study was tested in a cross-sectional dataset and an $\mathrm{AD}$ longitudinal dataset, and should be further validated with pharmacokinetic data, longitudinal data, and testretest analysis. Fourth, because WM intensity between $A \beta+$ and $\mathrm{A} \beta$ - patients is different, there may also be different histogram distributions in the two groups. However, this aspect was not considered in the present study. We plan to optimize the PERSI method by adding algorithms for 
different groups in future studies. Fifth, the definition of the cutoff value and norm for WM quantification is critical for determining positive or negative results in clinical applications, although such work has not been carried out to date and warrants study in the future. Sixth, the limited age range, which confounded amyloid quantification performance, may be a limitation of the present study. Future studies may require other extended age data to fully verify the applicability of the PERSI method. Seventh, the dataset used in the present study were collected from the ADNI database; however, the database lacks detailed information on injected dose and body weight, which meant the SUVR test could not be assessed. In future studies, we will test the generalization of SUVR using our own dataset. Finally, PERSI may entail the sacrifice of additional processing compared with standard reference regions to integral to its role as an accurate amyloid quantification in individual. Future studies may improve the PERSI method.

\section{Conclusions}

Our findings suggest that the PERSI method can provide accurate and robust count normalization for ${ }^{18} \mathrm{~F}-\mathrm{AV}-45$ images semi-quantitatively and can track $\mathrm{A} \beta$ pathophysiological processes in AD. This method can provide accurate characterization of subjects with or without AD continuum. Therefore, the PERSI method could have potential clinical application in nuclear medicine.

\section{Acknowledgments}

Funding: The present study was supported by grants from the National Natural Science Foundation of China (No. 61603236, 81671239, 81361120393, 81401135, 81771483, 81601454, 81830059, and 91849126), and the National Key R\&D Program of China (No. 2018YFC1314700). Data collection and sharing for this project was funded by the Alzheimer's Disease Neuroimaging Initiative (ADNI) (National Institutes of Health Grant U01 AG024904) and DOD ADNI (Department of Defense award number W81XWH-12-2-0012). ADNI is funded by the National Institute on Aging, the National Institute of Biomedical Imaging and Bioengineering, and through generous contributions from AbbVie, Alzheimer's Association, Alzheimer's Drug Discovery Foundation, Araclon Biotech, BioClinica, Biogen, Bristol-Myers Squibb, CereSpir, Cogstate, Eisai, Elan Pharmaceuticals, Eli Lilly and Company, EuroImmun, F. Hoffmann-La Roche and its affiliated company Genentech, Fujirebio, GE Healthcare, IXICO, Janssen Alzheimer Immunotherapy Research \& Development LLC, Johnson \& Johnson Pharmaceutical Research \& Development LLC, Lumosity, Lundbeck, Merck \& Co., Meso Scale Diagnostics LLC, NeuroRx Research, Neurotrack Technologies, Novartis Pharmaceuticals, Pfizer, Piramal Imaging, Servier, Takeda Pharmaceutical, and Transition Therapeutics. The Canadian Institutes of Health Research provides funds to support ADNI clinical sites in Canada. Private sector contributions are facilitated by the Foundation for the National Institutes of Health (www.fnih.org). The grantee organization is the Northern California Institute for Research and Education, and the study was coordinated by the Alzheimer's Therapeutic Research Institute at the University of Southern California. ADNI data are disseminated by the Laboratory for Neuroimaging at the University of Southern California.

\section{Footnote}

Conflicts of Interest: All authors have completed the ICMJE uniform disclosure form (available at http://dx.doi. org/10.21037/qims-20-110). The authors have no conflicts of interest to declare.

Ethical Statement: The present study was approved by the Institutional Review Board of Huashan Hospital. Written informed consent was obtained from the ADNI database.

Open Access Statement: This is an Open Access article distributed in accordance with the Creative Commons Attribution-NonCommercial-NoDerivs 4.0 International License (CC BY-NC-ND 4.0), which permits the noncommercial replication and distribution of the article with the strict proviso that no changes or edits are made and the original work is properly cited (including links to both the formal publication through the relevant DOI and the license). See: https://creativecommons.org/licenses/by-nc-nd/4.0/.

\section{References}

1. Alzheimer's Association. 2018 Alzheimer's disease facts and figures. Alzheimer's \& Dementia 2018;14:367-429.

2. Braak H, Alafuzoff I, Arzberger T, Kretzschmar H, Del Tredici K. Staging of Alzheimer diseaseassociated neurofibrillary pathology using paraffin sections and immunocytochemistry. Acta Neuropathol 
2006;112:389-404.

3. Jagust $W$. Imaging the evolution and pathophysiology of Alzheimer disease. Nat Rev Neurosci 2018;19:687-700.

4. Pike KE, Savage G, Villemagne VL, Ng S, Moss SA, Maruff P, Mathis CA, Klunk WE, Masters CL, Rowe CC. Beta-amyloid imaging and memory in non-demented individuals: evidence for preclinical Alzheimer's disease. Brain 2007;130:2837-44.

5. Musiek ES, Holtzman DM. Origins of Alzheimer's disease: reconciling cerebrospinal fluid biomarker and neuropathology data regarding the temporal sequence of amyloid-beta and tau involvement. Curr Opin Neurol 2012;25:715-20.

6. Jack CR Jr, Knopman DS, Jagust WJ, Petersen RC, Weiner MW, Aisen PS, Shaw LM, Vemuri P, Wiste HJ, Weigand SD, Lesnick TG, Pankratz VS, Donohue MC, Trojanowski JQ. Tracking pathophysiological processes in Alzheimer's disease: an updated hypothetical model of dynamic biomarkers. Lancet Neurol 2013;12:207-16.

7. Humayun H, Yao J. Imaging the aged brain: pertinence and methods. Quant Imaging Med Surg 2019;9:842-57.

8. Jack CR Jr, Bennett DA, Blennow K, Carrillo MC, Dunn B, Haeberlein SB, Holtzman DM, Jagust W, Jessen F, Karlawish J, Liu E, Molinuevo JL, Montine T, Phelps C, Rankin KP, Rowe CC, Scheltens P, Siemers E, Snyder HM, Sperling R. NIA-AA Research Framework: Toward a biological definition of Alzheimer's disease. Alzheimers Dement 2018;14:535-62.

9. Mathis CA, Wang Y, Holt DP, Huang GF, Debnath ML, Klunk WE. Synthesis and evaluation of 11C-labeled 6-substituted 2-arylbenzothiazoles as amyloid imaging agents. J Med Chem 2003;46:2740-54.

10. Choi SR, Golding G, Zhuang Z, Zhang W, Lim N, Hefti F, Benedum TE, Kilbourn MR, Skovronsky D, Kung HF. Preclinical properties of 18F-AV-45: a PET agent for Abeta plaques in the brain. J Nucl Med 2009;50:1887-94.

11. Koole M, Lewis DM, Buckley C, Nelissen N, Vandenbulcke M, Brooks DJ, Vandenberghe R, Van Laere $\mathrm{K}$. Whole-body biodistribution and radiation dosimetry of 18F-GE067: a radioligand for in vivo brain amyloid imaging. J Nucl Med 2009;50:818-22.

12. Shooli H, Dadgar H, Wáng YJ, Vafaee MS, Kashuk SR, Nemati R, Jafari E, Nabipour I, Gholamrezanezhad A, Assadi M, Larvie M. An update on PET-based molecular imaging in neuro-oncology: challenges and implementation for a precision medicine approach in cancer care. Quant Imaging Med Surg 2019;9:1597-610.

13. Ottoy J, Verhaeghe J, Niemantsverdriet E, Wyffels L,
Somers C, De Roeck E, Struyfs H, Soetewey F, Deleye S, Van den Bossche T, Van Mossevelde S, Ceyssens S, Versijpt J, Stroobants S, Engelborghs S, Staelens S. Validation of the Semiquantitative Static SUVR Method for (18)F-AV45 PET by Pharmacokinetic Modeling with an Arterial Input Function. J Nucl Med 2017;58:1483-9.

14. Lin KJ, Hsiao IT, Hsu JL, Huang CC, Huang KL, Hsieh CJ, Wey SP, Yen TC. Imaging characteristic of dualphase (18)F-florbetapir (AV-45/Amyvid) PET for the concomitant detection of perfusion deficits and betaamyloid deposition in Alzheimer's disease and mild cognitive impairment. Eur J Nucl Med Mol Imaging 2016;43:1304-14.

15. Chen K, Roontiva A, Thiyyagura P, Lee W, Liu X, Ayutyanont N, Protas H, Luo JL, Bauer R, Reschke C, Bandy D, Koeppe RA, Fleisher AS, Caselli RJ, Landau S, Jagust WJ, Weiner MW, Reiman EM. Improved power for characterizing longitudinal amyloid-beta PET changes and evaluating amyloid-modifying treatments with a cerebral white matter reference region. J Nucl Med 2015;56:560-6.

16. Landau SM, Fero A, Baker SL, Koeppe R, Mintun M, Chen K, Reiman EM, Jagust WJ. Measurement of longitudinal $\beta$-amyloid change with $18 \mathrm{~F}$-florbetapir PET and standardized uptake value ratios. J Nucl Med 2015;56:567-74.

17. Becker GA, Ichise M, Barthel H, Luthardt J, Patt M, Seese A, Schultze-Mosgau M, Rohde B, Gertz HJ, Reininger C, Sabri O. PET quantification of $18 \mathrm{~F}$-florbetaben binding to beta-amyloid deposits in human brains. J Nucl Med 2013;54:723-31.

18. Wong KP, Wardak M, Shao W, Dahlbom M, Kepe V, Liu J, Satyamurthy N, Small GW, Barrio JR, Huang SC. Quantitative analysis of [18F]FDDNP PET using subcortical white matter as reference region. Eur J Nucl Med Mol Imaging 2010;37:575-88.

19. Brendel M, Hogenauer M, Delker A, Sauerbeck J, Bartenstein P, Seibyl J, Rominger A. Improved longitudinal [(18)F]-AV45 amyloid PET by white matter reference and VOI-based partial volume effect correction. Neuroimage 2015;108:450-9.

20. Thomas BA, Erlandsson K, Modat M, Thurfjell L, Vandenberghe R, Ourselin S, Hutton BF. The importance of appropriate partial volume correction for PET quantification in Alzheimer's disease. Eur J Nucl Med Mol Imaging 2011;38:1104-19.

21. Erlandsson K, Buvat I, Pretorius PH, Thomas BA, Hutton BF. A review of partial volume correction techniques for emission tomography and their applications in neurology, 
cardiology and oncology. Phys Med Biol 2012;57:R119-59.

22. Gonzalez-Escamilla G, Lange C, Teipel S, Buchert R, Grothe MJ. PETPVE12: an SPM toolbox for Partial Volume Effects correction in brain PET - Application to amyloid imaging with AV45-PET. Neuroimage 2017;147:669-77.

23. Southekal S, Devous MD Sr, Kennedy I, Navitsky M, Lu M, Joshi AD, Pontecorvo MJ, Mintun MA. Flortaucipir F 18 Quantitation Using Parametric Estimation of Reference Signal Intensity. J Nucl Med 2018;59:944-51.

24. Müller-Gärtner HW, Links JM, Prince JL, Bryan RN, McVeigh E, Leal JP, Davatzikos C, Frost JJ. Measurement of radiotracer concentration in brain gray matter using positron emission tomography: MRI-based correction for partial volume effects. J Cereb Blood Flow Metab 1992;12:571-83.

25. Rousset OG, Ma Y, Evans AC. Correction for partial volume effects in PET: principle and validation. J Nucl Med 1998;39:904-11.

26. Petersen RC, Aisen PS, Beckett LA, Donohue MC, Gamst AC, Harvey DJ, Jack CR Jr, Jagust WJ, Shaw LM, Toga AW, Trojanowski JQ, Weiner MW. Alzheimer's Disease Neuroimaging Initiative (ADNI): clinical characterization. Neurology 2010;74:201-9.

27. Mazziotta J, Toga A, Evans A, Fox P, Lancaster J, Zilles K, Woods R, Paus T, Simpson G, Pike B, Holmes C, Collins L, Thompson P, MacDonald D, Iacoboni M, Schormann T, Amunts K, Palomero-Gallagher N, Geyer S, Parsons L, Narr K, Kabani N, Le Goualher G, Boomsma D, Cannon T, Kawashima R, Mazoyer B. A probabilistic atlas and reference system for the human brain: International Consortium for Brain Mapping (ICBM). Philos Trans R Soc Lond B Biol Sci 2001;356:1293-322.

28. Tzourio-Mazoyer N, Landeau B, Papathanassiou D, Crivello F, Etard O, Delcroix N, Mazoyer B, Joliot M. Automated anatomical labeling of activations in SPM using a macroscopic anatomical parcellation of the MNI MRI single-subject brain. Neuroimage 2002;15:273-89.

29. Hsiao IT, Huang CC, Hsieh CJ, Wey SP, Kung MP, Yen TC, Lin KJ. Perfusion-like template and standardized normalization-based brain image analysis using 18F-florbetapir (AV-45/Amyvid) PET. Eur J Nucl Med Mol Imaging 2013;40:908-20.

30. Xia M, Wang J, He Y. BrainNet Viewer: a network visualization tool for human brain connectomics. PLoS One 2013;8:e68910.

31. Rinne JO, Brooks DJ, Rossor MN, Fox NC, Bullock R, Klunk WE, Mathis CA, Blennow K, Barakos J, Okello
AA, Rodriguez Martinez de Liano S, Liu E, Koller M, Gregg KM, Schenk D, Black R, Grundman M. 11C-PiB PET assessment of change in fibrillar amyloidbeta load in patients with Alzheimer's disease treated with bapineuzumab: a phase 2, double-blind, placebocontrolled, ascending-dose study. Lancet Neurol 2010;9:363-72.

32. Leinonen V, Alafuzoff I, Aalto S, Suotunen T, Savolainen S, Nagren K, Tapiola T, Pirttila T, Rinne J, Jaaskelainen JE, Soininen H, Rinne JO. Assessment of beta-amyloid in a frontal cortical brain biopsy specimen and by positron emission tomography with carbon 11-labeled Pittsburgh Compound B. Arch Neurol 2008;65:1304-9.

33. van Berckel BN, Ossenkoppele R, Tolboom N, Yaqub M, Foster-Dingley JC, Windhorst AD, Scheltens P, Lammertsma AA, Boellaard R. Longitudinal amyloid imaging using 11C-PiB: methodologic considerations. J Nucl Med 2013;54:1570-6.

34. Doraiswamy PM, Sperling RA, Coleman RE, Johnson KA, Reiman EM, Davis MD, Grundman M, Sabbagh MN, Sadowsky CH, Fleisher AS, Carpenter A, Clark CM, Joshi AD, Mintun MA, Skovronsky DM, Pontecorvo MJ. Amyloid- $\beta$ assessed by florbetapir F 18 PET and 18-month cognitive decline: a multicenter study. Neurology 2012;79:1636-44.

35. Fleisher AS, Chen K, Liu X, Ayutyanont N, Roontiva A, Thiyyagura P, Protas H, Joshi AD, Sabbagh M, Sadowsky CH, Sperling RA, Clark CM, Mintun MA, Pontecorvo MJ, Coleman RE, Doraiswamy PM, Johnson KA, Carpenter AP, Skovronsky DM, Reiman EM. Apolipoprotein E $\varepsilon 4$ and age effects on florbetapir positron emission tomography in healthy aging and Alzheimer disease. Neurobiol Aging 2013;34:1-12.

36. Landau SM, Breault C, Joshi AD, Pontecorvo M, Mathis CA, Jagust WJ, Mintun MA. Amyloid- $\beta$ imaging with Pittsburgh compound B and florbetapir: comparing radiotracers and quantification methods. J Nucl Med 2013;54:70-7.

37. Cho H, Choi JY, Hwang MS, Kim YJ, Lee HM, Lee HS, Lee JH, Ryu YH, Lee MS, Lyoo CH. In vivo cortical spreading pattern of tau and amyloid in the Alzheimer disease spectrum. Ann Neurol 2016;80:247-58.

38. Shokouhi S, McKay JW, Baker SL, Kang H, Brill AB, Gwirtsman HE, Riddle WR, Claassen DO, Rogers BP. Reference tissue normalization in longitudinal (18) F-florbetapir positron emission tomography of late mild cognitive impairment. Alzheimers Res Ther 2016;8:2.

39. Rullmann M, Dukart J, Hoffmann KT, Luthardt J, 
Tiepolt S, Patt M, Gertz HJ, Schroeter ML, Seibyl J, Schulz-Schaeffer WJ, Sabri O, Barthel H. Partial-Volume Effect Correction Improves Quantitative Analysis of 18F-Florbetaben beta-Amyloid PET Scans. J Nucl Med 2016;57:198-203.

40. Chen YJ, Rosario BL, Mowrey W, Laymon CM, Lu X, Lopez OL, Klunk WE, Lopresti BJ, Mathis CA, Price JC. Relative 11C-PiB Delivery as a Proxy of Relative CBF: Quantitative Evaluation Using Single-Session 15O-Water and 11C-PiB PET. J Nucl Med 2015;56:1199-205.

41. Johnson KA, Sperling RA, Gidicsin CM, Carmasin JS, Maye JE, Coleman RE, Reiman EM, Sabbagh MN, Sadowsky CH, Fleisher AS, Murali Doraiswamy P,

Cite this article as: Wang $M$, Yan Z, Zhang $\mathrm{H}$, Lu J, Li L, Yu J, Wang J, Matsuda H, Zuo C, Jiang J; the Alzheimer's Disease Neuroimaging Initiative. Parametric estimation of reference signal intensity in the quantification of amyloidbeta deposition: an ${ }^{18} \mathrm{~F}-\mathrm{AV}-45$ study. Quant Imaging Med Surg 2021;11(1):249-263. doi: /10.21037/qims-20-110
Carpenter AP, Clark CM, Joshi AD, Lu M, Grundman M, Mintun MA, Pontecorvo MJ, Skovronsky DM. Florbetapir (F18-AV-45) PET to assess amyloid burden in Alzheimer's disease dementia, mild cognitive impairment, and normal aging. Alzheimers Dement 2013;9:S72-83.

42. Duan H, Jiang J, Xu J, Zhou H, Huang Z, Yu Z, Yan Z. Differences in Abeta brain networks in Alzheimer's disease and healthy controls. Brain Res 2017;1655:77-89.

43. Guo S, Xiao B, Wu C. Identifying subtypes of mild cognitive impairment from healthy aging based on multiple cortical features combined with volumetric measurements of the hippocampal subfields. Quant Imaging Med Surg 2020;10:1477-89. 


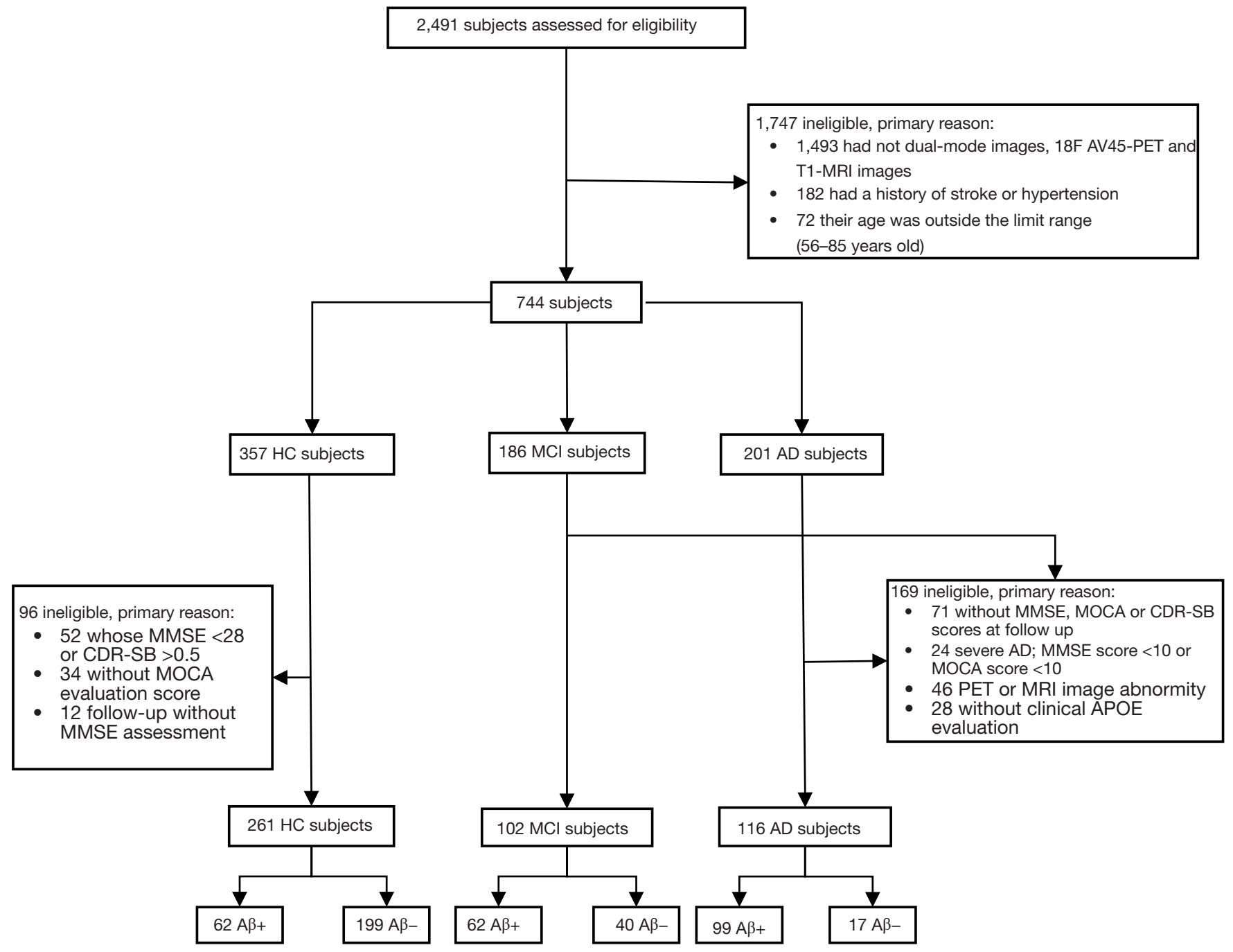

Figure S1 Trial profile of all study participants. A $\beta$, amyloid-beta; AD, Alzheimer's disease; APOE, apolipoprotein E; CDR-SB, Clinical Dementia Rating-Sum of Boxes; HC, healthy control; MCI, mild cognitive impairment; MOCA, Montreal Cognitive Assessment; MMSE, Mini-Mental State Examination; PET, positron emission tomography; ${ }^{18} \mathrm{~F}-\mathrm{AV}-45$, florbetapir; +, positive; -, negative. 
Table S1 Clinical ratings and demographic characteristics of longitudinal AD participants

\begin{tabular}{lccc}
\hline \multirow{2}{*}{ Characteristics } & & AD $(n=14)$ & P value \\
\cline { 2 - 4 } Sex (male/female) & $A \beta$ positive $(n=13)$ & A negative $(n=1)$ & $1 / 0$ \\
Baseline age (years) & $9 / 4$ & 81 & - \\
Follow-up duration (years) & $74.0 \pm 5.6$ & 2 & - \\
Education (years) & $2.23 \pm 0.83$ & 14 & - \\
MMSE & $16.5 \pm 3.2$ & -3 & - \\
MOCA & $-3.38 \pm 3.52^{*}$ & 1 & - \\
CDR-SB & $-5.23 \pm 3.78^{*}$ & 5 & - \\
APOE $\varepsilon 4$ positivity (\%) & $2.15 \pm 2.23^{*}$ & 0 & \\
\hline
\end{tabular}

${ }^{*}, \mathrm{P}<0.05$, 1-sample $t$-test. $A \beta$, amyloid-beta; AD, Alzheimer's disease; APOE $\varepsilon 4$ positivity, positive rate for the presence of at least $1 \varepsilon 4$ apolipoprotein E; CDR-SB, Clinical Dementia Rating-Sum of Boxes; MOCA, Montreal Cognitive Assessment; MMSE, Mini-Mental State Examination.

Table S2 Diagnostic ability of 4 semi-quantitative methods in the frontal lobe

\begin{tabular}{|c|c|c|c|c|c|}
\hline Characteristics & Group & Whole cerebellum & MNI atlas-based WM & Subject-specific WM & PERSI-WM \\
\hline \multirow{4}{*}{ SUVR (frontal lobe) } & $\mathrm{MCl} A \beta+$ & $1.48 \pm 0.15$ & $0.72 \pm 0.08$ & $0.70 \pm 0.08$ & $0.71 \pm 0.07$ \\
\hline & $A D A \beta+$ & $1.49 \pm 0.12$ & $0.72 \pm 0.06$ & $0.71 \pm 0.06$ & $0.79 \pm 0.07$ \\
\hline & $\mathrm{HCA} \beta+$ & $1.40 \pm 0.12$ & $0.63 \pm 0.07$ & $0.61 \pm 0.07$ & $0.62 \pm 0.06$ \\
\hline & $A D A \beta-$ & $1.13 \pm 0.07$ & $0.56 \pm 0.09$ & $0.56 \pm 0.09$ & $0.68 \pm 0.10$ \\
\hline \multirow[t]{3}{*}{ AUC } & $\mathrm{AD} \& \mathrm{MCl} A \beta+v s . \mathrm{HC} A \beta-$ & 0.975 & 0.961 & 0.966 & 0.975 \\
\hline & $A D A \beta+$ vs. $H C A \beta-$ & 0.979 & 0.967 & 0.975 & 0.989 \\
\hline & $\mathrm{MCl} A \beta+$ vs. $\mathrm{HC} A \beta-$ & 0.969 & 0.952 & 0.951 & 0.955 \\
\hline Effect size & $\mathrm{MCl} A \beta+$ vs. HC A $\beta-$ & 2.27 & 2.18 & 2.11 & 2.28 \\
\hline
\end{tabular}

$\mathrm{A} \beta$, amyloid-beta; $\mathrm{AD}$, Alzheimer's disease; $\mathrm{AUC}$, area under the curve, $\mathrm{HC}$, healthy control; $\mathrm{MCl}$, mild cognitive impairment; $\mathrm{MNI}$, Montreal Neurological Institute; PERSI, parametric estimation of reference signal intensity; SUVR, standardized uptake value ratio; WM, white matter; +, positive; -, negative. 
Table S3 Diagnostic ability of 4 semi-quantitative methods in the parietal lobe

\begin{tabular}{|c|c|c|c|c|c|}
\hline Characteristics & Group & Whole cerebellum & MNI atlas-based WM & Subject-specific WM & PERSI-WM \\
\hline \multirow{4}{*}{ SUVR (parietal lobe) } & $\mathrm{MCl} \mathrm{A} \beta+$ & $1.44 \pm 0.16$ & $0.70 \pm 0.08$ & $0.68 \pm 0.08$ & $0.69 \pm 0.07$ \\
\hline & $A D A \beta+$ & $1.42 \pm 0.14$ & $0.68 \pm 0.06$ & $0.68 \pm 0.06$ & $0.75 \pm 0.07$ \\
\hline & $\mathrm{HCA} \beta+$ & $1.37 \pm 0.13$ & $0.62 \pm 0.07$ & $0.60 \pm 0.07$ & $0.60 \pm 0.07$ \\
\hline & $A D A \beta-$ & $1.08 \pm 0.07$ & $0.54 \pm 0.08$ & $0.54 \pm 0.08$ & $0.65 \pm 0.10$ \\
\hline \multirow[t]{3}{*}{ AUC } & $A D \& M C I A \beta+v s . H C A \beta-$ & 0.965 & 0.948 & 0.955 & 0.971 \\
\hline & $A D A \beta+v s . H C A \beta-$ & 0.956 & 0.946 & 0.958 & 0.983 \\
\hline & $\mathrm{MCl} A \beta+$ vs. $\mathrm{HC} A \beta-$ & 0.978 & 0.949 & 0.949 & 0.951 \\
\hline Effect size & $\mathrm{MCl} A \beta+$ vs. $\mathrm{HC} \mathrm{A} \beta-$ & 2.27 & 2.00 & 1.95 & 2.07 \\
\hline
\end{tabular}

$\mathrm{A} \beta$, amyloid-beta; $\mathrm{AD}$, Alzheimer's disease; $\mathrm{AUC}$, area under the curve, $\mathrm{HC}$, healthy control; $\mathrm{MCl}$, mild cognitive impairment; $\mathrm{MNI}$, Montreal Neurological Institute; PERSI, parametric estimation of reference signal intensity; SUVR, standardized uptake value ratio; WM, white matter; +, positive; -, negative.

Table S4 Diagnostic ability of 4 semi-quantitative methods in the temporal lobe

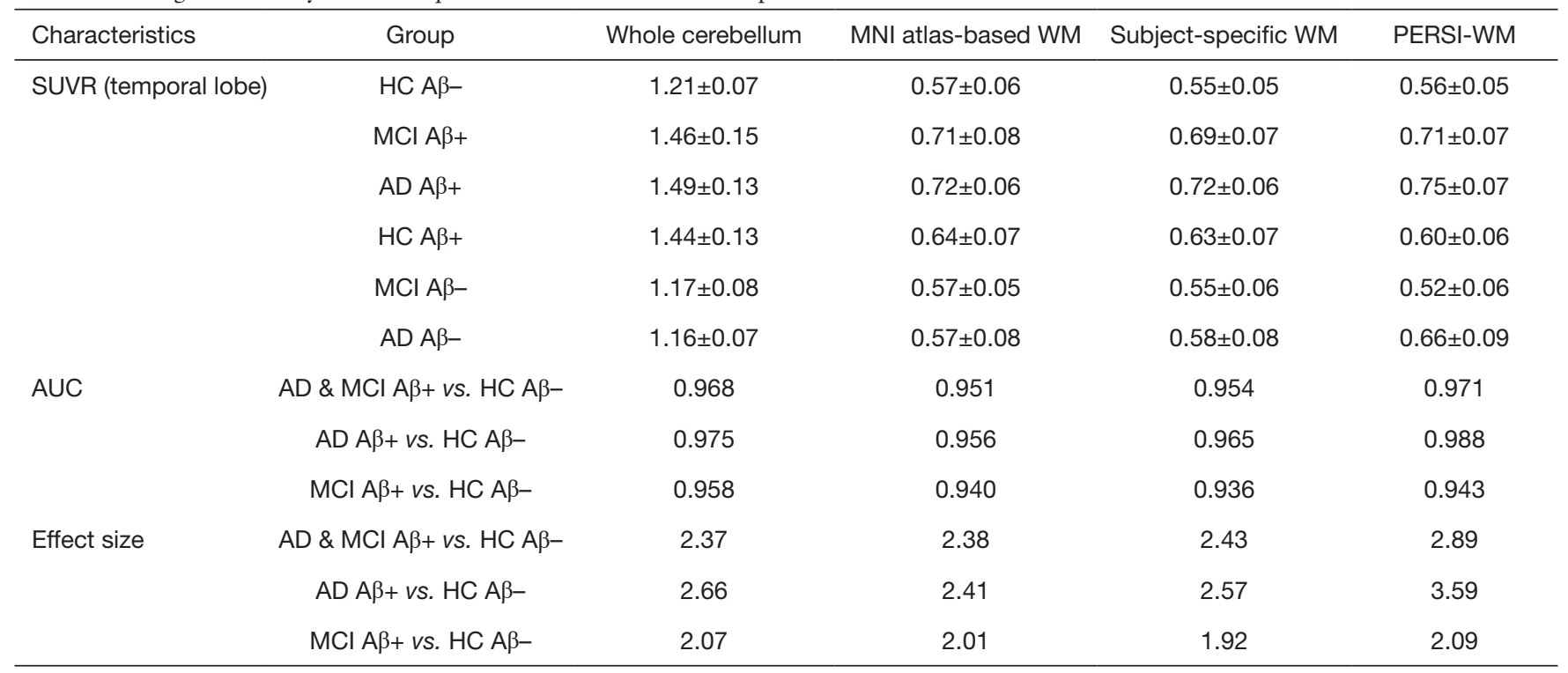

$A \beta$, amyloid-beta; $A D$, Alzheimer's disease; AUC, area under the curve, $\mathrm{HC}$, healthy control; $\mathrm{MCl}$, mild cognitive impairment; MNI, Montreal Neurological Institute; PERSI, parametric estimation of reference signal intensity; SUVR, standardized uptake value ratio; WM, white matter; +, positive; -, negative. 
Table S5 Diagnostic ability of 4 semi-quantitative methods in the occipital lobe

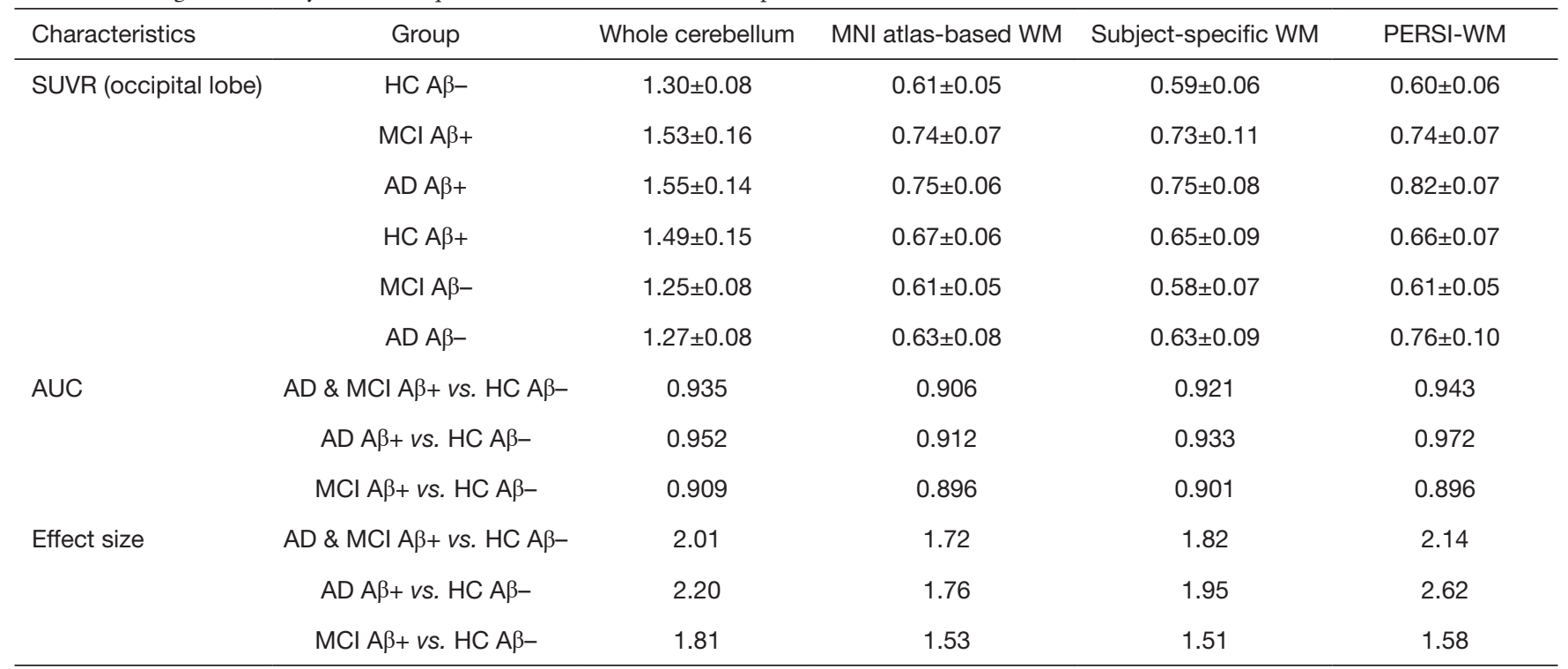

$\mathrm{A} \beta$, amyloid-beta; $\mathrm{AD}$, Alzheimer's disease; $\mathrm{AUC}$, area under the curve, $\mathrm{HC}$, healthy control; $\mathrm{MCl}$, mild cognitive impairment; $\mathrm{MNI}$, Montreal Neurological Institute; PERSI, parametric estimation of reference signal intensity; SUVR, standardized uptake value ratio; WM, white matter; +, positive; -, negative.

Table S6 Diagnostic ability of 4 semi-quantitative methods in the anterior cingulate

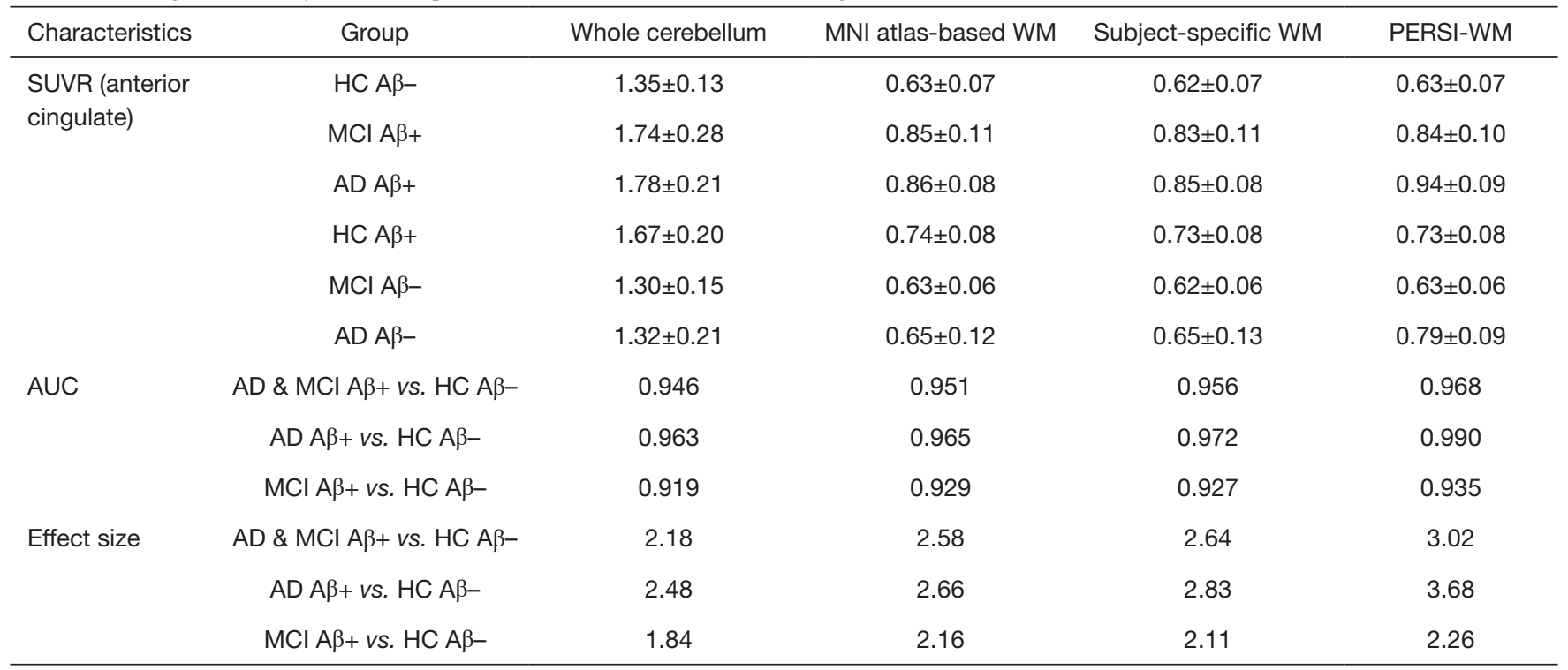

$\mathrm{A} \beta$, amyloid-beta; $\mathrm{AD}$, Alzheimer's disease; $\mathrm{AUC}$, area under the curve, $\mathrm{HC}$, healthy control; $\mathrm{MCl}$, mild cognitive impairment; $\mathrm{MNI}$, Montreal Neurological Institute; PERSI, parametric estimation of reference signal intensity; SUVR, standardized uptake value ratio; WM, white matter; +, positive; -, negative. 
Table S7 Diagnostic ability of 4 semi-quantitative methods in the posterior cingulate

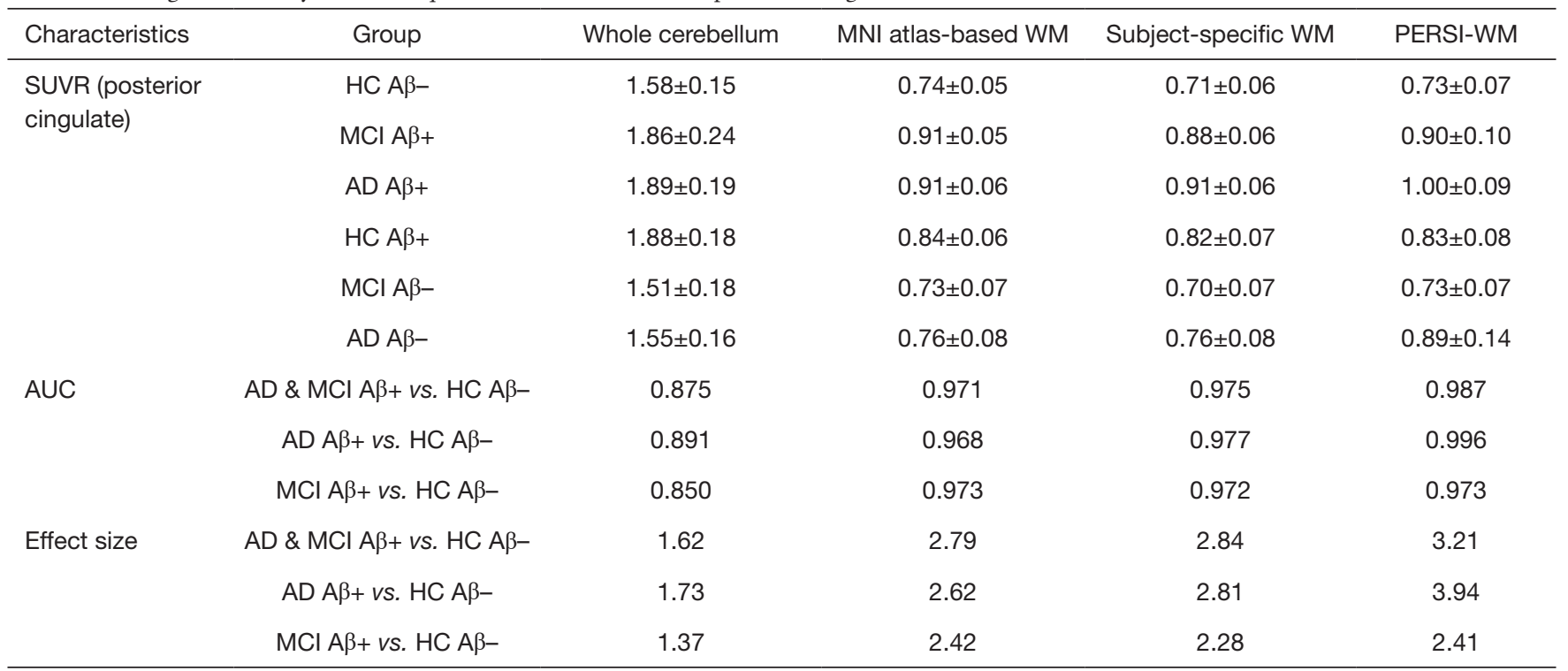

$\mathrm{A} \beta$, amyloid-beta; $\mathrm{AD}$, Alzheimer's disease; $\mathrm{AUC}$, area under the curve, $\mathrm{HC}$, healthy control; $\mathrm{MCl}$, mild cognitive impairment; $\mathrm{MNI}$, Montreal Neurological Institute; PERSI, parametric estimation of reference signal intensity; SUVR, standardized uptake value ratio; WM, white matter; +, positive; -, negative.

Table S8 Diagnostic ability of 4 semi-quantitative methods in the precuneus

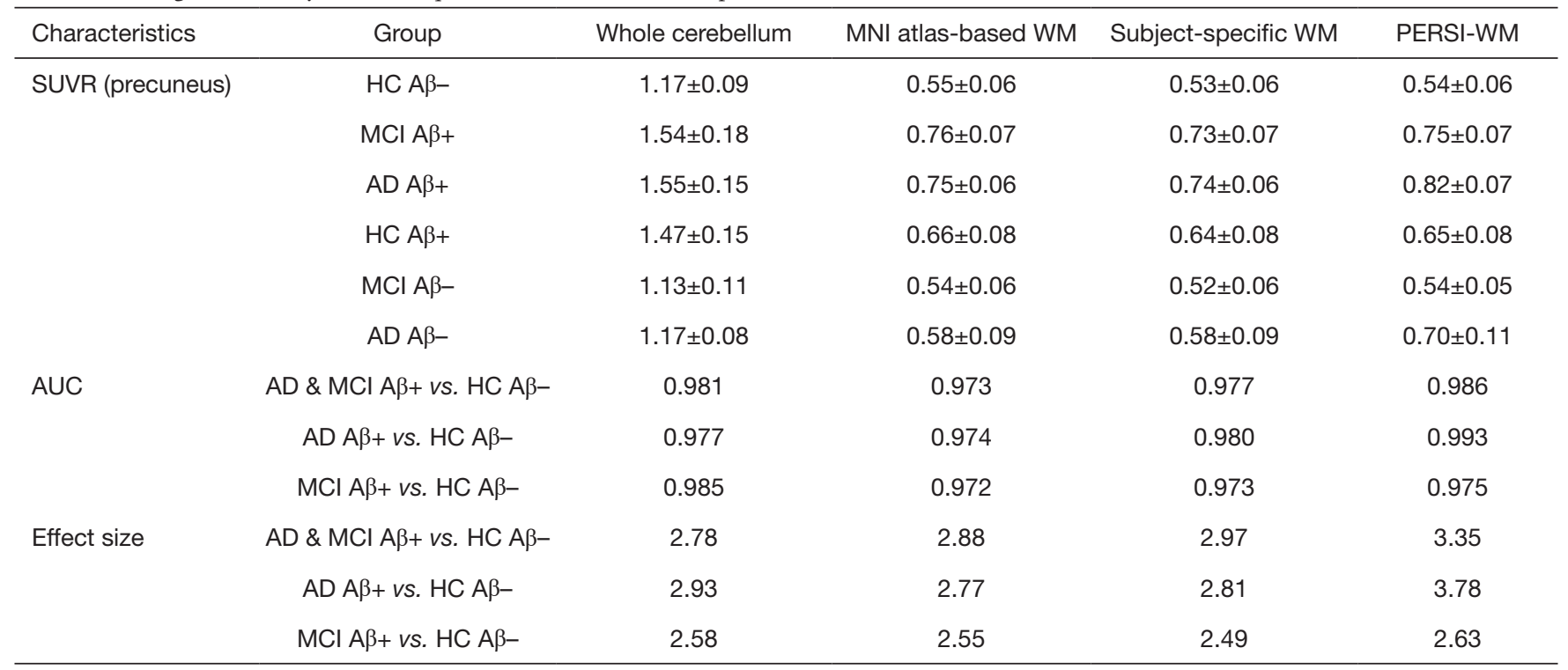

$\mathrm{A} \beta$, amyloid-beta; $\mathrm{AD}$, Alzheimer's disease; $\mathrm{AUC}$, area under the curve, $\mathrm{HC}$, healthy control; $\mathrm{MCl}$, mild cognitive impairment; $\mathrm{MNI}$, Montreal Neurological Institute; PERSI, parametric estimation of reference signal intensity; SUVR, standardized uptake value ratio; WM, white matter; +, positive; -, negative. 

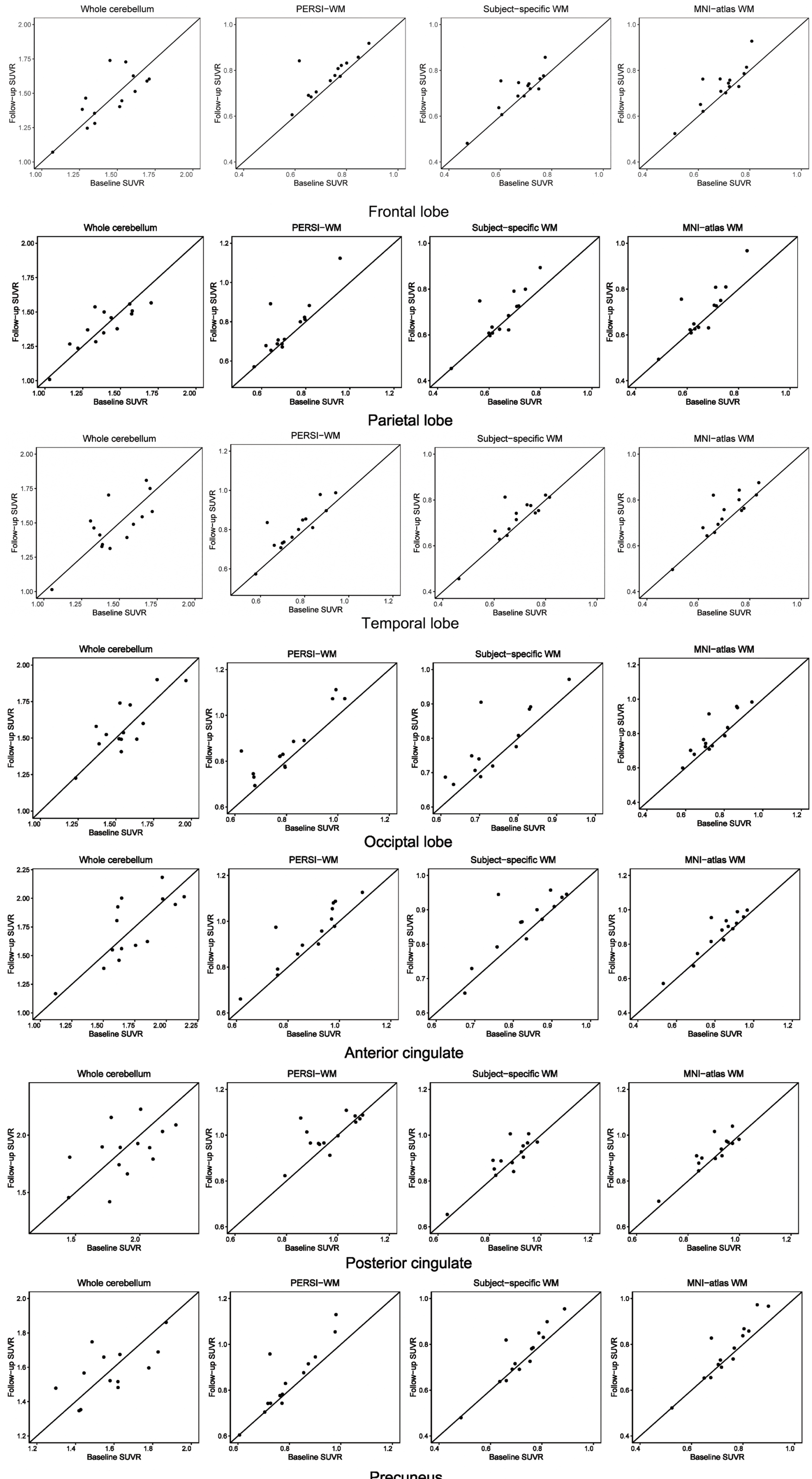

Figure S2 Fewer individual variations in tracking of increase in candidate regions of interest standardized uptake value ratios (SUVRs) with parametric estimation of reference signal intensity-white matter (PERSI-WM) than with other reference regions. MNI, Montreal Neurological Institute. 
Table S9 Correlations between clinical cognitive scores and standardized uptake value ratio of the frontal lobe

\begin{tabular}{lcccc}
\hline Frontal lobe & PERSI-WM & Subject-specific WM & MNI atlas-based WM & Whole cerebellum \\
\hline MMSE & -0.426 & -0.325 & -0.298 & $-0.144^{\dagger}$ \\
MOCA & -0.373 & -0.271 & -0.256 & $-0.093^{\dagger}$ \\
CDR-SB & 0.447 & 0.403 & 0.397 & 0.241 \\
\hline
\end{tabular}

${ }^{\dagger}$, insignificant correlation, $\mathrm{P}$ value with correction for multiple comparisons >0.05. CDR-SB, Clinical Dementia Rating-Sum of Boxes; MMSE, Mini-Mental State Examination; MNI, Montreal Neurological Institute; MOCA, Montreal Cognitive Assessment; PERSI, parametric estimation of reference signal intensity; WM, white matter.

Table S10 Correlations between clinical cognitive scores and standardized uptake value ratio of the parietal lobe

\begin{tabular}{lcccc}
\hline Parietal lobe & PERSI-WM & Subject-specific WM & MNI atlas-based WM & Whole cerebellum \\
\hline MMSE & -0.387 & -0.294 & -0.243 & $-0.124^{\dagger}$ \\
MOCA & -0.355 & -0.248 & -0.231 & $-0.078^{\dagger}$ \\
CDR-SB & 0.437 & 0.383 & 0.375 & 0.221 \\
\hline
\end{tabular}

${ }^{\dagger}$, insignificant correlation, $\mathrm{P}$ value with correction for multiple comparisons $>0.05$. CDR-SB, Clinical Dementia Rating-Sum of Boxes; MMSE, Mini-Mental State Examination; MNI, Montreal Neurological Institute; MOCA, Montreal Cognitive Assessment; PERSI, parametric estimation of reference signal intensity; WM, white matter.

Table S11 Correlations between clinical cognitive scores and standardized uptake value ratio of the temporal lobe

\begin{tabular}{lcccc}
\hline Temporal lobe & PERSI-WM & Subject-specific WM & MNI atlas-based WM & Whole cerebellum \\
\hline MMSE & -0.415 & -0.317 & -0.291 & $-0.134^{\dagger}$ \\
MOCA & -0.364 & -0.263 & -0.243 & $-0.007^{\dagger}$ \\
CDR-SB & 0.402 & 0.347 & 0.338 & $0.187^{\dagger}$ \\
\hline
\end{tabular}

${ }^{\dagger}$, insignificant correlation, $\mathrm{P}$ value with correction for multiple comparisons $>0.05$. CDR-SB, Clinical Dementia Rating-Sum of Boxes; MMSE, Mini-Mental State Examination; MNI, Montreal Neurological Institute; MOCA, Montreal Cognitive Assessment; PERSI, parametric estimation of reference signal intensity; WM, white matter.

Table S12 Correlations between clinical cognitive scores and standardized uptake value ratio of the occipital lobe

\begin{tabular}{lcccc}
\hline Occipital lobe & PERSI-WM & Subject-specific WM & MNI atlas-based WM & Whole cerebellum \\
\hline MMSE & -0.454 & -0.372 & -0.339 & -0.217 \\
MOCA & -0.409 & -0.309 & -0.288 & $-0.127^{\dagger}$ \\
CDR-SB & 0.482 & 0.422 & 0.406 & 0.294 \\
\hline
\end{tabular}

${ }^{\dagger}$, insignificant correlation, $\mathrm{P}$ value with correction for multiple comparisons $>0.05$. CDR-SB, Clinical Dementia Rating-Sum of Boxes; MMSE, Mini-Mental State Examination; MNI, Montreal Neurological Institute; MOCA, Montreal Cognitive Assessment; PERSI, parametric estimation of reference signal intensity; WM, white matter. 
Table S13 Correlations between clinical cognitive scores and standardized uptake value ratio of the anterior cingulate

\begin{tabular}{lcccc}
\hline Anterior cingulate & PERSI-WM & Subject-specific WM & MNI atlas-based WM & Whole cerebellum \\
\hline MMSE & -0.402 & -0.302 & -0.281 & $-0.154^{\dagger}$ \\
MOCA & -0.356 & -0.268 & -0.255 & $-0.114^{\dagger}$ \\
CDR-SB & 0.398 & 0.355 & 0.346 & 0.229 \\
\hline
\end{tabular}

${ }^{\dagger}$, insignificant correlation, $\mathrm{P}$ value with correction for multiple comparisons $>0.05$. CDR-SB, Clinical Dementia Rating-Sum of Boxes; MMSE, Mini-Mental State Examination; MNI, Montreal Neurological Institute; MOCA, Montreal Cognitive Assessment; PERSI, parametric estimation of reference signal intensity; WM, white matter.

Table S14 Correlations between clinical cognitive scores and standardized uptake value ratio of the posterior cingulate

\begin{tabular}{lcccc}
\hline Posterior cingulate & PERSI-WM & Subject-specific WM & MNI atlas-based WM & Whole cerebellum \\
\hline MMSE & -0.338 & -0.209 & -0.175 & $-0.012^{\dagger}$ \\
MOCA & -0.341 & -0.223 & -0.199 & $-0.05^{\dagger}$ \\
CDR-SB & 0.368 & 0.271 & 0.255 & $0.08^{\dagger}$ \\
\hline
\end{tabular}

${ }^{\dagger}$, insignificant correlation, $\mathrm{P}$ value with correction for multiple comparisons $>0.05$. CDR-SB, Clinical Dementia Rating-Sum of Boxes; MMSE, Mini-Mental State Examination; MNI, Montreal Neurological Institute; MOCA, Montreal Cognitive Assessment; PERSI, parametric estimation of reference signal intensity; WM, white matter.

Table S15 Correlations between clinical cognitive scores and standardized uptake value ratio of the precuneus

\begin{tabular}{lcccc}
\hline Precuneus & PERSI-WM & Subject-specific WM & MNI atlas-based WM & Whole cerebellum \\
\hline MMSE & -0.394 & -0.295 & -0.273 & $-0.131^{\dagger}$ \\
MOCA & -0.356 & -0.247 & -0.231 & $-0.09^{\dagger}$ \\
CDR-SB & 0.427 & 0.382 & 0.375 & 0.227 \\
\hline
\end{tabular}

${ }^{\dagger}$, insignificant correlation, $\mathrm{P}$ value with correction for multiple comparisons $>0.05$. CDR-SB, Clinical Dementia Rating-Sum of Boxes; MMSE, Mini-Mental State Examination; MNI, Montreal Neurological Institute; MOCA, Montreal Cognitive Assessment; PERSI, parametric estimation of reference signal intensity; WM, white matter. 